\title{
Localized structures and front propagation in the Lengyel-Epstein model
}

\author{
Jensen, O.; Pannbacker, Viggo Ole; Mosekilde, Erik; Dewel, G.; Borckmans, P.
}

Published in:

Physical Review E. Statistical, Nonlinear, and Soft Matter Physics

Link to article, DOI:

10.1103/PhysRevE.50.736

Publication date:

1994

Document Version

Publisher's PDF, also known as Version of record

Link back to DTU Orbit

Citation (APA):

Jensen, O., Pannbacker, V. O., Mosekilde, E., Dewel, G., \& Borckmans, P. (1994). Localized structures and front propagation in the Lengyel-Epstein model. Physical Review E. Statistical, Nonlinear, and Soft Matter Physics, 50(2), 736-749. https://doi.org/10.1103/PhysRevE.50.736

\section{General rights}

Copyright and moral rights for the publications made accessible in the public portal are retained by the authors and/or other copyright owners and it is a condition of accessing publications that users recognise and abide by the legal requirements associated with these rights.

- Users may download and print one copy of any publication from the public portal for the purpose of private study or research.

- You may not further distribute the material or use it for any profit-making activity or commercial gain

- You may freely distribute the URL identifying the publication in the public portal 


\title{
Localized structures and front propagation in the Lengyel-Epstein model
}

\author{
O. Jensen, ${ }^{1}$ V. O. Pannbacker, ${ }^{1}$ E. Mosekilde, ${ }^{1}$ G. Dewel, ${ }^{2}$ and P. Borckmans ${ }^{2}$ \\ ${ }^{1}$ Physics Department, Technical University of Denmark, 2800 Lyngby, Denmark \\ ${ }^{2}$ Service de Chimie-Physique, Code Postal 231 Université Libre de Bruxelles, 1050 Brussels, Belgium
}

(Received 28 December 1993)

\begin{abstract}
Pattern selection, localized structure formation, and front propagation are analyzed within the framework of a model for the chlorine dioxide-iodine-malonic acid reaction that represents a key to understanding recently obtained Turing structures. This model is distinguished from previously studied, simple reaction-diffusion models by producing a strongly subcritical transition to stripes. The wave number for the modes of maximum linear gain is calculated and compared with the dominant wave number for the finally selected, stationary structures grown from the homogeneous steady state or developed behind a traveling front. The speed of propagation for a front between the homogeneous steady state and a one-dimensional (1D) Turing structure is obtained. This velocity shows a characteristic change in behavior at the crossover between the subcritical and supercritical regimes for the Turing bifurcation. In the subcritical regime there is an interval where the front velocity vanishes as a result of a pinning of the front to the underlying structure. In 2D, two different nucleation mechanisms for hexagonal structures are illustrated on the Lengyel-Epstein and the Brusselator model. Finally, the observation of 1D and 2D spirals with Turing-induced cores is reported.
\end{abstract}

PACS number(s): 64.60.Ht, 05.70.Ln

\section{INTRODUCTION}

In his classic paper on "The Chemical Basis of Morphogenesis," Turing [1] suggested that chemical reaction-diffusion systems under certain conditions could become unstable and yield to the formation of stationary, periodic concentration patterns. During the subsequent period of more than 40 years many aspects of Turing's theory have been worked out in detail [2-5], and a variety of different applications to biological pattern and form formation processes have been proposed [6-8].

Like other self-organizing structures [2,9-12], Turing patterns emerge spontaneously from a uniform background without any specific interference from the outside. They distinguish themselves by the active role that diffusion plays in destabilizing the chemical reaction. In contrast to patterns formed by many hydrodynamical instabilities, they are characterized by having an intrinsic wavelength, determined by the parameters of the reaction-diffusion process rather than by the size of the reactor. As opposed to Liesegang rings [13], only a single phase is involved, and no precipitation takes place.

Turing's suggestion stands as one of the most influential ideas in theoretical biology. Until recently, however, it has not been possible to obtain standing diffusion generated patterns for any reaction under laboratory conditions. To avoid disturbances associated with the supply of reactants, most previous work has been based on reactor designs representing chemical pool conditions, and the observed spirals and waves $[4,14]$ have neither been stationary in space nor generated by the Turing mechanism.

During the past few years a number of new reactor designs have been developed $[15,16]$ in which the reaction takes place in a thin layer of a transparent, chemically in- ert gel. This allows open system conditions to be maintained without the disturbing influence of convective flows. The gel is fed on either side from reservoirs where the chemical composition is controlled through continuous stirring and supply of fresh solutions. In the absence of any instability in the gel, the concentrations of reactants in the planes perpendicular to the feeding gradients will be uniform. However, when the reaction-diffusion instability arises, this symmetry is broken, and spatial patterns develop.

The unambiguous observation of Turing structures under laboratory conditions was first made by Castets $e t$ al. [17]. Experimenting with the chlorite-iodide-malonic acid (or so-called CIMA) reaction, they discovered the formation of standing three-dimensional (3D) structures with characteristic wavelengths of $0.2 \mathrm{~mm}$. The structures were confined to the central region of the reactor by the gradients in feed concentrations, and they could be maintained for $20 \mathrm{~h}$ or more, i.e., as long as the gel remained structurally stable. Subsequent studies by Ouyang and Swinney [18] using a somewhat different reactor geometry showed extended quasi-twodimensional patterns in the form of hexagons and stripes and also lead to the first experimental determination of the bifurcation diagram for the Turing instability.

As pointed out by Lengyel and Epstein [19], Turing structures could arise in these experiments because starch was used as a color indicator for the process. Starch forms a reversible complex with iodine, and the fact that this complex is practically immobile in the gel causes a significant reduction in the effective diffusion constant for iodide, hence creating the conditions of short range activation and long range inhibition required for the Turing mechanism to become active [20]. If the concentration of starch is sufficiently reduced, the stationary structures are 
found to disappear to give way to oscillations and waves [21]. In the transition region, a wealth of complex spatiotemporal behaviors show up, apparently as a result of an interaction between Turing and Hopf modes [22].

By using spectroscopic techniques, Lengyel, Rábai, and Epstein $[23,24]$ have been able to follow the concentrations of various intermediates and products of the CIMA reaction and to determine the corresponding rate constants. According to these measurements, the oscillations are related with a set of subsidiary reactions involving (i) the iodination of malonic acid, (ii) the oxidation of iodide ions by free chlorine dioxide radicals, and (iii) a reaction between chlorite and iodide ions to regenerate iodine.

In principle, the rate equations for this so-called CDIMA reaction involve five difference species. However, while the concentrations of chlorite and iodide ions experimentally are found to vary over several orders of magnitude during an oscillation, the concentrations of malonic acid, chlorine dioxide, and iodine show little wave-periodic variation. To a first approach, these concentrations may therefore be treated as constants, and, after proper rescaling, the dynamics of the system may be approximated by the following two-variable model [19]:

$$
\frac{\partial u}{\partial t}=a-u-\frac{4 u v}{1+u^{2}}+\nabla^{2} u
$$

and

$$
\frac{\partial v}{\partial t}=\delta\left[b\left[u-\frac{u v}{1+u^{2}}\right]+c \nabla^{2} v\right] .
$$

Here, $u$ denotes the concentration of iodide ions and $v$ the concentration of chorite ions. $a$ and $b$ are parameters that are related to the feed concentrations and to experimentally determined rate constants. A shift towards higher values of $a$ represents an increase in the supply of malonic acid relative to the supply of chlorine dioxide, and increasing $b$ corresponds to a higher supply of iodine. In a practical experiment, these concentrations are typically of the order of $3 \times 10^{-3}-3 \times 10^{-2} \mathrm{~mol} / 1$. $c$ is the ratio of the diffusion constant of chlorite to that of uncomplexed iodide, and $\delta$ is a rescaling parameter that depends upon the concentration of starch. The higher this concentration is, the larger $\delta$ will be. The nonlinear term $u v /\left(1+u^{2}\right)$ represents the self-inhibitory effect of $I^{-}$on the chlorite-iodide reaction [19].

The observation of Turing structures in the CIMA reaction has sparked a significant interest into the search for other pattern forming reactions [25]. Much experimental effort is also devoted to exposing in more detail the role played by the complexing agent and the gel matrix. In parallel with this there is a need to extend the theoretical analysis of the relation between kinetics and the kinds of patterns produced. We need to better understand the significance of multistability, localized structures, and front propagation, and we also need a better description of the dynamics of defects and the generation of chemical turbulence.

For systems with Brusselator-type kinetics, some of these problems have been dealt with in recent contribu- tions by De Wit et al. [26], Borckmans, De Wit, and Dewel [27], Verdasca et al. [28], and Dufiet and Boissonade $[29,30]$. In particular, De Wit et al. [26] have considered the full three-dimensional pattern selection problem corresponding to the experimental conditions realized by Castets et al. [17]. For systems with Lengyel-Epstein kinetics, preliminary results on structure formation and front propagation have been reported by Jensen et al. [31] and by Pannbacker et al. [32]. Numerically calculated bifurcation diagrams for this model show that the Turing bifurcation to stripes is strongly subcritical. This makes the model qualitatively different from other simple reaction-diffusion models capable of producing Turing structures, and a number of characteristic differences with previous results can thus be expected.

The present paper examines some of these phenomena in more detail. The gain curve, which relates the rate of linear growth for space-periodic structures to their wave number, is calculated from a linear stability analysis. A comparison of the wave number for the modes of maximum linear gain with those of the finally selected, stationary structures shows good agreement, provided that the pattern has developed from the homogeneous steady state. A pattern formed behind a traveling front, on the other hand, has a significantly longer wavelength. The speed of propagation for a front connecting the Turing pattern to the homogeneous steady state is calculated and compared to numerical results. Due to the linearization involved, the analytical results are valid only well into the supercritical regime. A comparison with numerical results shows a characteristic deviation as the bifurcation point is approached. In the subcritical regime there is a band of parameter values for which the front velocity vanishes as a result of pinning between the propagating front and the underlying structure. Within this band, a variety of different stationary and localized structures can arise. A similar phenomenon cannot be observed in the case of a supercritical bifurcation to stripes. Characteristic differences also arise in two-dimensional problems when considering the growth of a localized hexagonal structure into the surrounding homogeneous steady state. Finally, we present results on the observation in numerical simulations of $1 \mathrm{D}$ and $2 \mathrm{D}$ spirals with Turing-induced cores.

\section{PATTERN SELECTION}

Reaction-diffusion systems with autocatalytic or crosscatalytic processes are inherently nonlinear, and one can expect several stationary solutions to exist. Pattern selection is then to be viewed as an outcome of the interaction and competition processes among the various spatial modes that start to grow as the threshold for instability is crossed. This outcome clearly depends on the parameters that control the growth rates of the modes, on the initial and boundary conditions, and on the relative stability of the various stationary solutions. Just beyond the threshold of instability all modes with the critical wave number are equally amplified, independent on their orientation. This follows from the rotational invariance for large isotropic systems as those characterizing the recent experi- 
ments with the CIMA reaction. Because of this infinite degeneracy, the pattern that finally emerges cannot be determined solely by a linear stability analysis of the reference state. Further above the threshold, modes covering a relatively broad spectrum of different wave numbers become linearly unstable, and we are faced with the problem of wavelength selection. This is a very complex problem which to our knowledge has not yet found a general solution. A main line of approach is to consider the restrictions on the band of possible Turing modes brought on by so-called sideband instabilities (e.g., Eckhaus and zigzag instabilities for stripes). In 2D one also has to take patterns of hexagonal symmetry into account, and in 3D body centered cubic structures may appear.

Nonequilibrium systems often exhibit the phenomenon of multistability, i.e., the existence of more than one stable state for a given set of parameter values. The state that is selected in a specific experiment then depends on the history of the system preparation. In contrast to equilibrium phase transitions, no organizing principle exists that predicts a unique stationary solution. This allows for structures to arise and be stable that have no counterpart in equilibrium systems. It is also well established that fluctuations generally play a minor role in chemical instabilities. The transitions between the different states then take place at the marginal stability points via the decay of an unstable mode contrary to most equilibrium phase transitions.

Self-organizing processes exhibit universal features that depend solely on the symmetry properties of the system. It is nevertheless important to keep in mind that the parameters of the specific problem determine how the possible structures compete and interact with one another and hence the rate at which one structure invades another. Moreover, the universality is soon lost as the process proceeds through secondary and tertiary bifurcations, and one is led to consider scenarios leading eventually to the complicated spatiotemporal patterns that can arise in large aspect-ratio systems.

To start the analysis let us first consider the results of a linear approach. As described by the Lengyel-Epstein model Eqs. (1) and (2), the CDIMA reaction is of activator-inhibitor type with $u$ as the activator and $v$ the inhibitor. Moreover, the effective diffusion constant $\delta c$ for the inhibitor is much larger than the diffusion constant for the activator as required for Turing structures to arise [2]. The model exhibits a unique steady state at $\left(u_{0}, v_{0}\right)=\left(a / 5,1+a^{2} / 25\right)$. This state is independent of $b$, and it is therefore practical to use $b$ as the bifurcation parameter in the following calculations. If diffusion is neglected, the homogeneous steady state is stable as long as $b>b_{H}=\left(3 a^{2}-5^{3}\right) / 5 a \delta$ where a Hopf bifurcation occurs. When diffusion is taken into account, a new linear stability analysis shows that the system becomes unstable towards standing perturbations of finite wave number for [32]

$$
b<b_{T}=\frac{\left[5^{3}+13 a^{2}-4 a \sqrt{10\left(5^{2}+a^{2}\right)}\right] c}{5 a} .
$$

$b_{T}$ is independent of $\delta$. On the other hand, $b_{H}$ varies inversely with $\delta$, and for sufficiently high concentration of starch, $b_{T}>b_{H}$. Hence, an interval exists in which the homogeneous steady state is stable towards uniform oscillations, but unstable with respect to the growth of standing patterns of finite wavelength. In one dimension, the finally selected stationary patterns are stripes. In two dimensions they may be stripes or hexagons, while in three dimensions the first structure to appear has body centered cubic symmetry [26]. A calculation of the gain characteristic, i.e., the relation between the growth rate of the pattern and its wave number in the small signal approximation, gives

$$
\lambda_{r}(k)=\frac{1}{2} \operatorname{Re}\left\{-m\left(k^{2}\right)+\sqrt{m^{2}\left(k^{2}\right)-4 h\left(k^{2}\right)}\right\}
$$

with

$$
m\left(k^{2}\right)=k^{2}(1+\delta c)-\operatorname{tr} A
$$

and

$$
h\left(k^{2}\right)=\delta c k^{4}-\left(\delta c f_{u}+g_{v}\right) k^{2}+|A| .
$$

Here, $\lambda_{r}(k)$ is the real part of the eigenvalue.

$$
f(u, v)=a-u-\frac{4 u v}{1+u^{2}}
$$

and

$$
g(u, v)=\delta b\left(u-\frac{u v}{1+u^{2}}\right)
$$

are the right-hand sides of the rate equations (1) and (2) without diffusion. $A$ is the corresponding Jacobian matrix evaluated at the equilibrium point, and $|A|$ is its determinant. The subscripts $u$ and $v$ in the expression for $h\left(k^{2}\right)$ denote differentiation with respect to each of these variables, respectively.

By evaluating (4) for $a=30, b=1.6, c=1.5$, and $\delta=8$ we obtain the gain curve shown in Fig. 1. As illustrated by this figure, there is a spectrum of modes with wave numbers between $k_{1}$ and $k_{2}$ for which the gain factor is

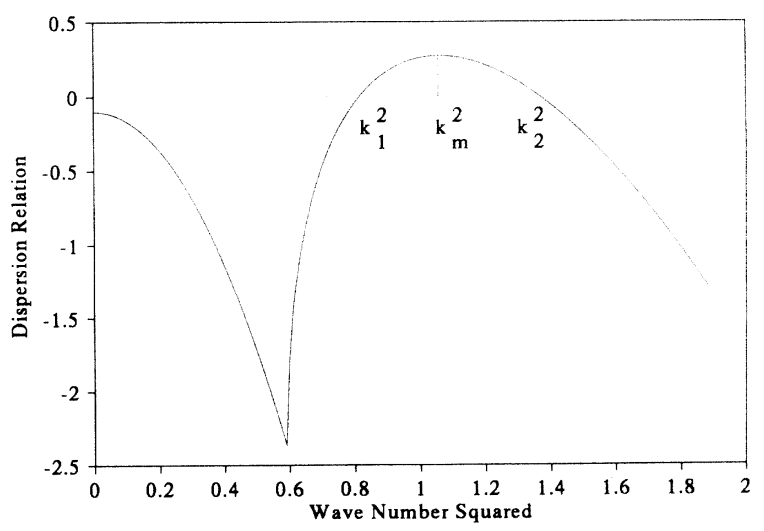

FIG. 1. Dispersion relation for the Lengyel-Epstein model showing the real part of the eigenvalue as a function of the (squared) wave number for standing, spatially periodic small signal perturbations. With the assumed parameters, only Turing modes with wave numbers $k_{1}>k>k_{2}$ are unstable. 
positive. There is another maximum of the gain curve around $k=0$. This maximum arises from the Hopf instability. However, with the assumed parameters, the Hopf modes have $\operatorname{Re}\{\lambda\}<0$, and the system is stable against homogeneous oscillations. A decrease in $b$ will shift the gain curve upwards, and the range of wave numbers for which amplification takes place will increase. The highest overall growth rate is obtained for $k=k_{m}$ where $\lambda_{r}(k)$ has a maximum. It is possible to obtain an analytical expression for $k_{m}$ in terms of the parameters of the model. We shall not reproduce this expression here. Instead, in Fig. 2 we have plotted (fully drawn smooth curve) the variation of $k_{m}$ with $b$ for $a=30, c=1.5$, and $\delta=8$. The wave number for the modes of maximum linear gain is seen to increase slowly with $b$ throughout the supercritical regime. The highest value of $b$ in the figure corresponds approximately to the Turing bifurcation point $b_{T}$. If absolute values are inserted for the various parameters, the range of wave numbers for which amplification can occur is found to agree well with the experimentally observed wavelengths around $0.2 \mathrm{~mm}$ [17].

Figure 3 shows the bifurcation diagram for the Lengyel-Epstein model with a single space dimension [31]. Starting with a value of $b$ in the interval for Turing instabilities $\left(b_{H}<b<b_{T}\right)$, this diagram was obtained by seeding the homogeneous steady state with noise and integrating the dynamical equations to obtain the final, stationary pattern. Hereafter, this pattern was used as initial conditions when restarting the integration with a slightly different value of $b$. With this adiabatic approach we could follow the stationary structure (and determine its amplitude and wave number) as a function of $b$ into the subcritical region $\left(b>b_{T}\right)$ where it cannot grow out of the noise seeded homogeneous steady state. The numerical calculations were performed by means of a semiimplicit Crank-Nicolson method with no-flux boundary

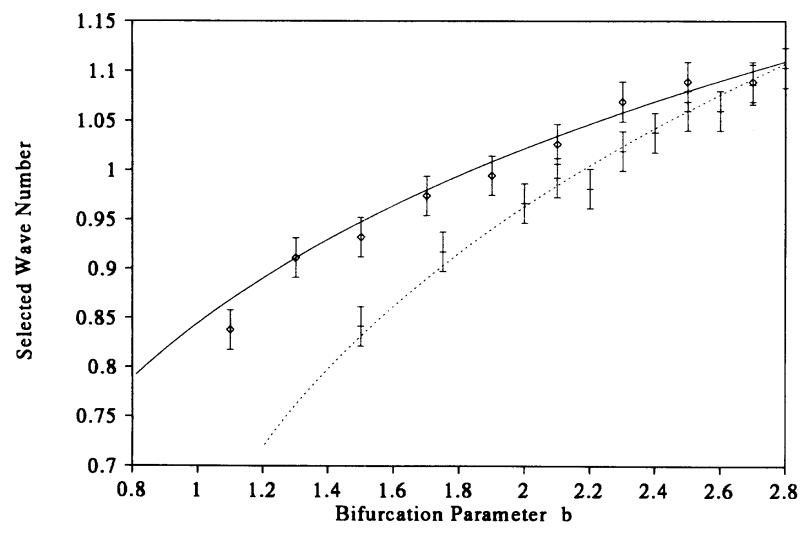

FIG. 2. Wave number selection in the Lengyel-Epstein model. The fully drawn curve shows the wave number for the modes of maximum linear gain. The dotted curve reproduces analytical results for the wave number selected behind a traveling front (see below). Diamonds and crosses are numerically determined results for the final stationary patterns developed from the homogeneous steady state and behind a traveling front, respectively.

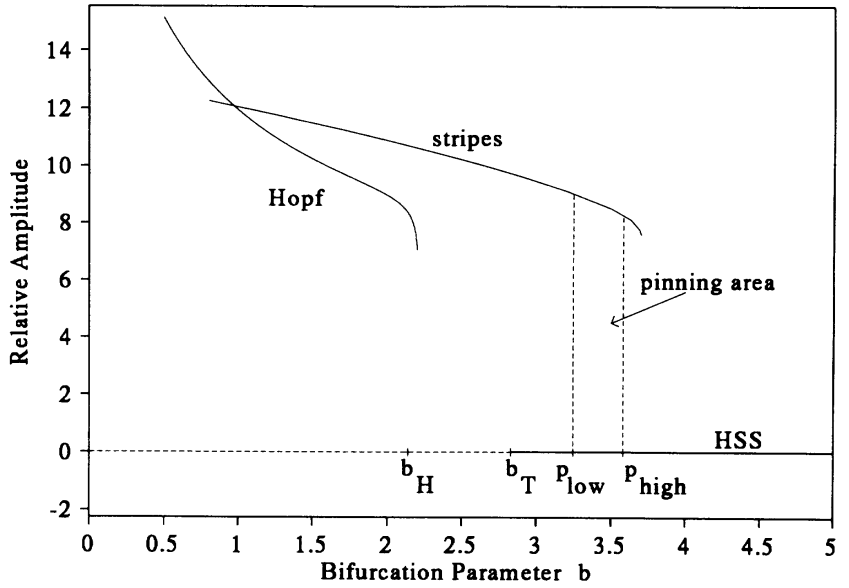

FIG. 3. Numerically calculated bifurcation diagram for a one-dimensional Lengyel-Epstein model with $a=30$. $c$ and $\delta$ attain their standard values of 1.5 and 8 , respectively. Note the regions of subcriticality for the two bifurcations and the region of bistability between Turing structures and Hopf oscillations. HSS denotes the homogeneous steady state.

conditions. In each simulation, the stability of the obtained structure was tested by applying a small noise signal. A similar approach was applied to determine the bifurcation curve for the Hopf oscillations. In the region $0.8<b<1.0$, where the Turing stripes have a lower amplitude than the Hopf oscillations, the linear stability analysis still shows that the Turing modes have the highest gain factors. When starting from a noise seeded homogeneous steady state one may then observe that the stripe pattern starts to develop and almost saturates. At this stage small regions of the structure start to oscillate, and after some time the system ends up in a uniformly oscillatory state with no spatial inhomogeneities.

With the assumed parameters $(a=30, c=1.5$, and $\delta=8$ ), the Hopf bifurcation is weakly inverted whereas the Turing bifurcation is highly subcritical. There is also a range where Hopf oscillations coexist with stationary stripes. The overlapping stability regions allow for a variety of different inhomogeneous solutions to arise. We shall return to this problem in the following section. First, however, let us try to compare the wave number for the numerically computed stationary pattern with the wave number $k_{m}$ for the modes of maximum linear gain. Such a comparison is provided in Fig. 2, where the diamonds with error bars represent the wave numbers of the stationary stripe structure obtained when starting each simulation from the homogeneous steady state. Note that in general this procedure will not give the same wavelength for the finally selected structures as the adiabatic approach used to construct the bifurcation diagram in Fig. 3. The agreement with the analytical results (fully drawn curve) is clearly quite good, indicating that the modes of maximum linear gain remain dominant also in the nonlinear region. The final structure is not sinusoidal, however, but contains a spectrum of higher harmonics not shown in the figure.

In two dimensions, the picture is even more complicated. Here, we have the possibility of both hexagons and 


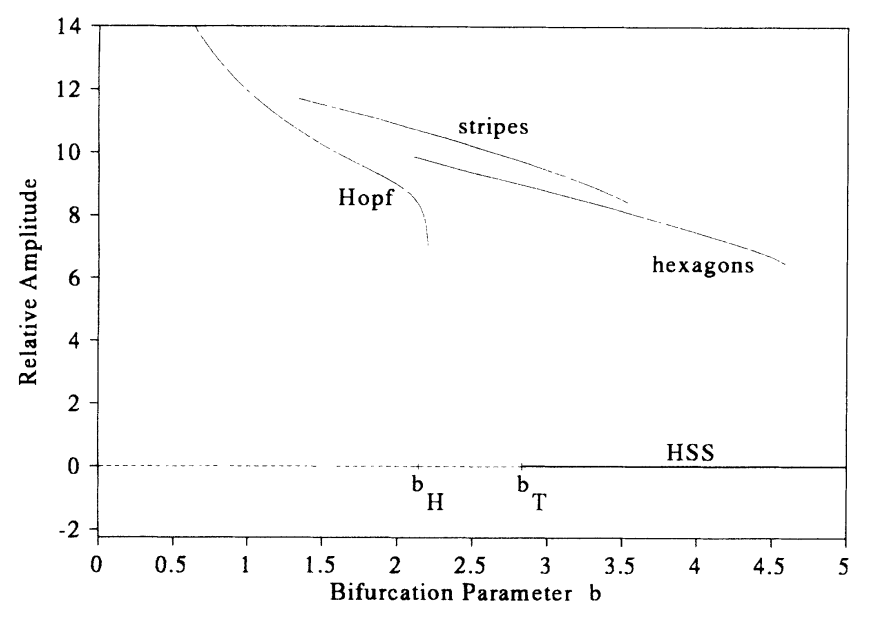

FIG. 4. Numerically calculated bifurcation diagram for the two-dimensional Lengyel-Epstein model. Note the region of tristability between hexagons, stripes, and the homogeneous steady state.

stripes, and both types of structures have been experimentally observed [18].

Moreover, for hexagons the phase is important, since the activator, for instance, can show either concentration maxima or minima in the centers of the hexagons. Accordingly, we talk about 0 - and $\pi$-hexagons. An additional problem of a more numerical character is that the applied boundary conditions tend to impose specific orientations on the obtained structures. Stripes, for instance, may tend to arrange themselves so that they are perpendicular to the boundaries [30]. In two dimensions, our calculations have been performed either with an alternating directions implicit method with no-flux boundary conditions or with an odd-even hopscotch method and periodic boundary conditions. Only the periodic boundary conditions are neutral regarding the orientation of the patterns.

With these modifications, the procedures for obtaining the two-dimensional bifurcation diagram depicted in Fig. 4 were similar to those described above for the onedimensional case. Several features of Fig. 4 are worth noticing. In particular, we see that the Turing bifurcations leading to stripes and hexagons are both subcritical. We also see that there is a significant region of overlap of the stability intervals for the two types of structures. When $b$ becomes large enough for the stripes to become unstable, a transition to hexagons may occur. By following this transition in time, one can see how the stripes break up and transform into hexagons. At the lower end of the stability interval for hexagons, on the other hand, one can observe a transition in which hexagons join hexagons to produce stripes.

\section{FRONT PROPAGATION IN 1D}

The strongly subcritical nature of the Turing bifurcation to stripes distinguishes the Lengyel-Epstein model from previously studied, simple reaction-diffusion models capable of showing this type of instability. This subcriticality provides the possibility of a variety of inhomogeneous solutions, some of which may be stable [31]. For certain parameters, for instance, we may find a stable stripe structure surrounded by regions of homogeneous steady state, or we may find an islet of homogeneous steady state in a sea of Turing stripes. The simplest inhomogeneous solution consists of a front (or kink) separating a region with stripes from the homogeneous steady state. This situation is illustrated in Fig. 5(a), which shows a stripe structure that invades the homogeneous steady state.
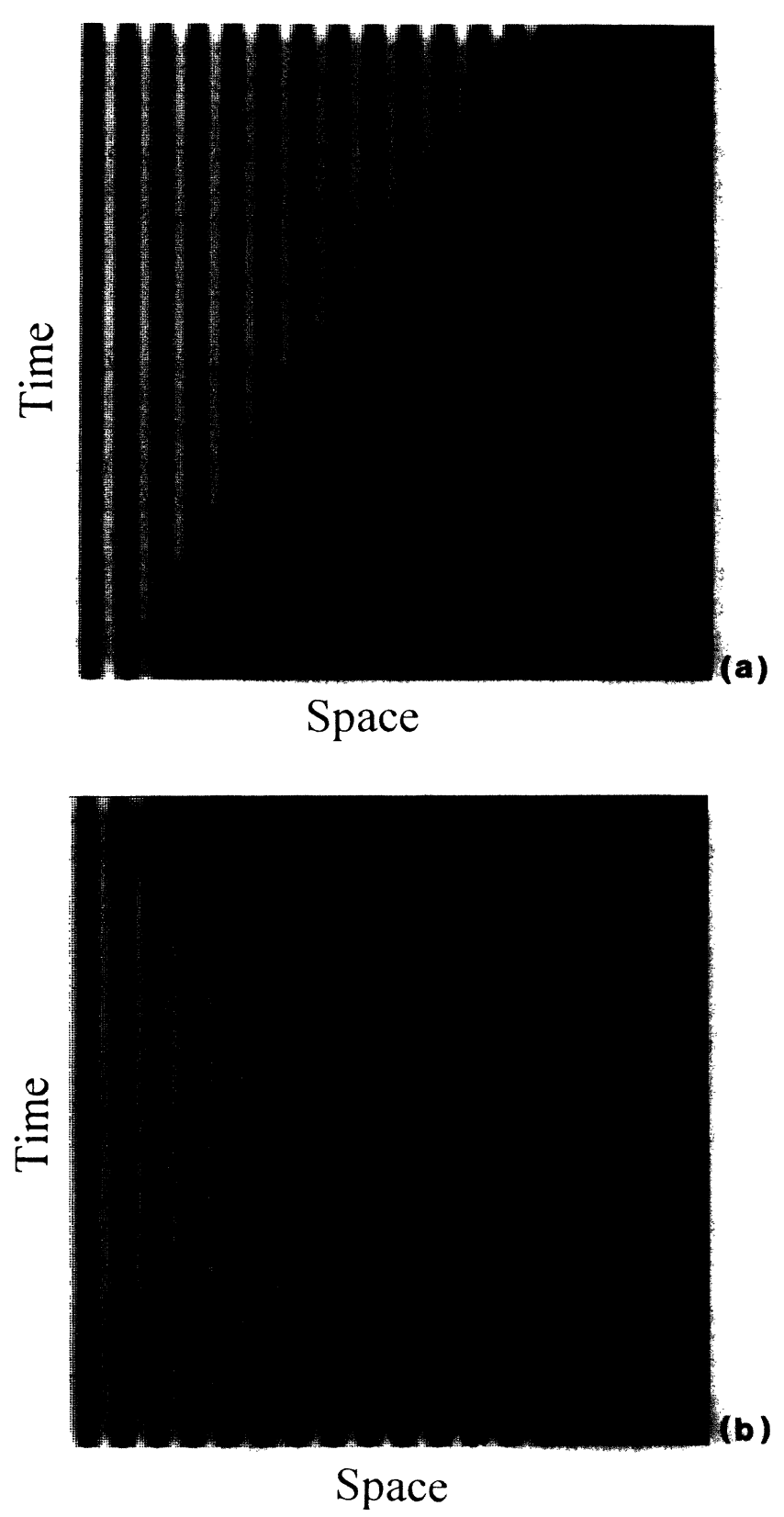

FIG. 5. Numerically computed solutions to the LengyelEpstein model with one space dimension: (a) a stripe structure invades the homogeneous steady state for $a=30$ and $b=3.0$, and (b) the homogeneous steady state invades a region with stripe structure for $a=30$ and $b=3.6$. 
This will occur in an interval below and immediately above the bifurcation point ( $b<p_{\text {low }}$ in Fig. 3). The opposite situation in which the homogeneous steady state invades a region with stripes is shown in Fig. 5(b). This will occur close to the upper end of the subcritical region $\left(p_{\text {high }}<b\right)$. Between these two regions there is a band ( $p_{\text {low }}<b<p_{\text {high }}$ ) in which the front velocity vanishes, and the two states can coexist in a stable manner.

To analyze the propagation of fronts in more detail, let us assume that we are well into the supercritical regime $\left(b<b_{T}\right)$, where only the Turing structure is stable. Particular initial conditions may have produced a situation in which a front exists between a region with stationary stripes and the unstable homogeneous steady state. Figure 6 illustrates a typical front profile.

Calculation of the front velocity may now proceed as follows [33-35]. The leading edge of the front, where small signal conditions apply, is assumed to be described as the product of an exponentially rising function $\phi_{\mathrm{env}}$ (the envelope) and a standing sinusoidal variation $\phi_{\text {osc }}$. As previously indicated, the solutions to the linearized system can be expressed as

$$
\phi(x, t) \cong e^{i k x+\lambda(k) t},
$$

where

$$
\lambda(k)=\frac{1}{2}\left[-m\left(k^{2}\right)+\sqrt{m^{2}\left(k^{2}\right)-4 h\left(k^{2}\right)}\right]
$$

with $m\left(k^{2}\right)$ and $h\left(k^{2}\right)$ as given by Eqs. (5) and (6). By separating real and imaginary parts $\left[k=k_{r}+i k_{i}\right.$, $\left.\lambda(k)=\lambda_{r}(k)+i \lambda_{i}(k)\right]$, Eq. (9) may be rewritten as

$$
\phi_{\mathrm{env}} \phi_{\mathrm{osc}} \cong e^{-k_{i} x+\lambda_{r}(k) t} e^{i\left[k_{r} x+\lambda_{i}(k) t\right]} .
$$

Here, we identify the nonoscillatory part as the envelope. If this envelope is to propagate with uniform velocity $v_{f}$ and unchanged shape, we must have (marginal stability hypothesis)

$$
v_{f}=\frac{\lambda_{r}(k)}{k_{i}} \text {. }
$$

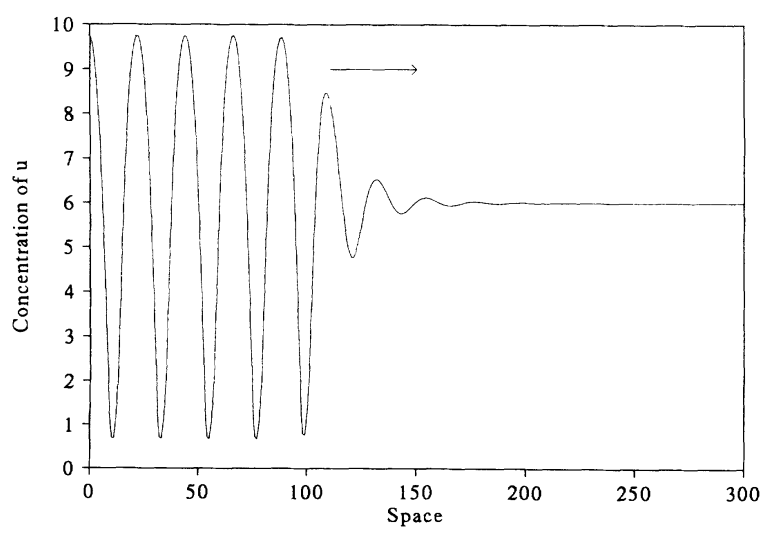

FIG. 6. Profile of a front between a region with stationary Turing stripes and the homogeneous steady state. The front propagates to the right as the Turing structure invades the unstable homogeneous steady state.
In a coordinate system following the front, the standing stripe pattern will appear as a temporal oscillation, and one oscillation in time will correspond to one period in space. The angular frequency evaluated in the moving frame is

$$
\omega=k_{r} v_{f}+\lambda_{i}(k) \text {. }
$$

Hence, as long as the number of nodes is conserved as they pass through the front, the wave number of the pattern behind the front must be

$$
k_{p}=\frac{\omega}{v_{f}}=k_{r}+\frac{\lambda_{i}(k)}{v_{f}} .
$$

These expressions alone cannot give us $v_{f}$ and $k_{p}$, since we do not know $k_{r}$ and $k_{i}$. However, if we impose the additional requirements that the front velocity $v_{f}$ be real and that the front itself be stable against any ( real or imaginary) small change in $k$, we obtain the additional conditions (stationary phase).

$$
v_{f}=-\frac{\partial \lambda_{i}(k)}{\partial k_{r}}
$$

and

$$
0=\frac{\partial \lambda_{r}(k)}{\partial k_{r}} .
$$

Together with the dispersion relation (10) these expressions suffice to determine the velocity of the front $v_{f}$ and the wave number $k_{p}$ of the modes selected behind the front. In practice, these calculations are fairly complicated and can best be performed by means of approximate methods. We have solved the problem by expanding $k$ around the value $k_{c}$ obtained for $k_{m}$ at the Turing bifurcation point where $k_{i}$ and $\lambda_{r}(k)$ vanish. The expansion was performed by means of symbolic manipulation (MATHEMATICA), and by choosing the order of expansion high enough we can obtain the results with the desired precision.

As calculated from the above analysis, $k_{p}$ may clearly differ from $k_{m}$. A comparison between the two wave numbers is provided in Fig. 2, where the dotted curve shows the variation of $k_{p}$ through the supercritical regime. At the bifurcation point $b \cong 2.8$, the two wave numbers coincide, $k_{p}$ and $k_{m}$ both being equal to $k_{c}=\sqrt{\left(\delta c f_{u}+g_{v}\right) / 2 \delta c}$. However, as we move into the supercritical regime, $k_{p}$ decreases faster than $k_{m}$, implying that a structure developed behind a traveling front will have a longer wavelength than a structure that has grown from the homogeneous steady state. The points with error bars around the dotted curve represent numerical results obtained for the fully developed stripe structure existing behind the front. Again it appears that the simple marginal stability theory is capable of predicting the characteristics of the stationary structure.

Figure 7 shows the variation of the front velocity with $b$. Here, the fully drawn curve represents the velocity $v_{f}$ as calculated from the above analytical approach, and the points with error bars represent numerically determined values for the front velocity obtained by integrating the 


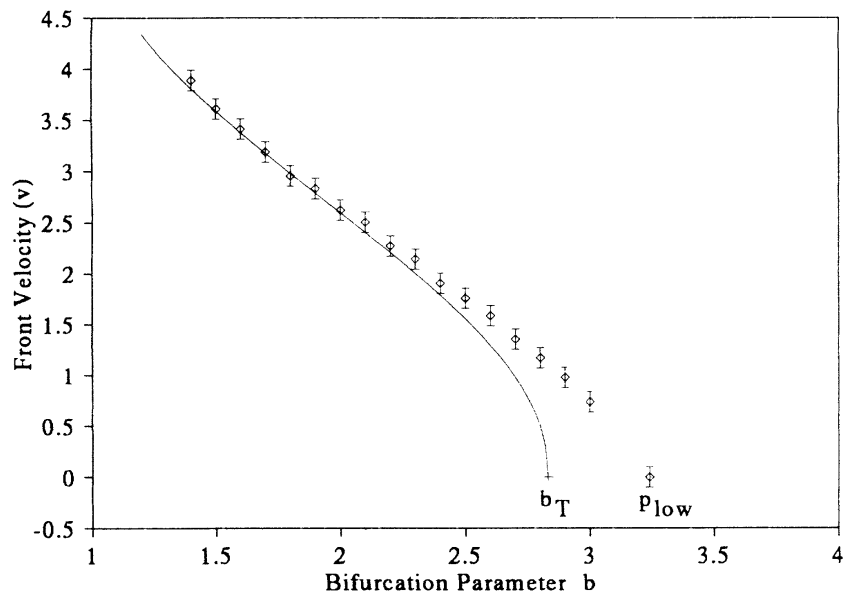

FIG. 7. Variation of the front velocity with the bifurcation parameter $b$ for a front separating Turing stripes from the homogeneous steady state. The fully drawn line represents the analytical results and the diamonds with error bars represent numerically obtained results.

one-dimensional Lengyel-Epstein model with initial conditions that produce a front. Well into the supercritical regime, the two calculations give similar results. However, as we approach the bifurcation point, the results start to differ. While the analytical results for $v_{f}$ approaches zero at $b=b_{T}$, the simulations show a characteristic crossover to a different curve which approaches zero at $b=p_{\text {low }}$. An analogous result has been found by van Saarloos and Hohenberg [36] in a study of front propagation in the complex Ginzburg-Landau equation, although in this case pinning effects are absent. In the crossover region the velocity of the front is not uniform [32]. Rather, the front jumps one wavelength at a time, and the interval between subsequent jumps increases as the pinning band is approached. Such interactions between a front and an underlying structure are frequent in solid state physics, where they are known to influence the dynamics of dislocations, charge density waves, and magnetic domain walls. We have performed a similar analysis for the classical Brusselator model [2],

$$
\begin{aligned}
& \frac{\partial x}{\partial t}=A-B x+x^{2} y-x+D_{x} \nabla^{2} x, \\
& \frac{\partial y}{\partial t}=B x-x^{2} y+D_{y} \nabla^{2} y,
\end{aligned}
$$

for which the Turing bifurcation to stripes is supercritical $[27,28]$. Figure 8 shows the results for the wave number selection as a function of $B$ for $A=30, D_{x}=1.5$, and $D_{y}=8$. Again, the fully drawn curve represents the analytical results for the modes of maximum linear gain, and the dotted curve represents the modes selected behind a propagating front according to the above analysis. The points with error bars represent numerically obtained wave numbers for the final stationary solutions emerging from the homogeneous steady state and developing behind a front, respectively. Again we ob-

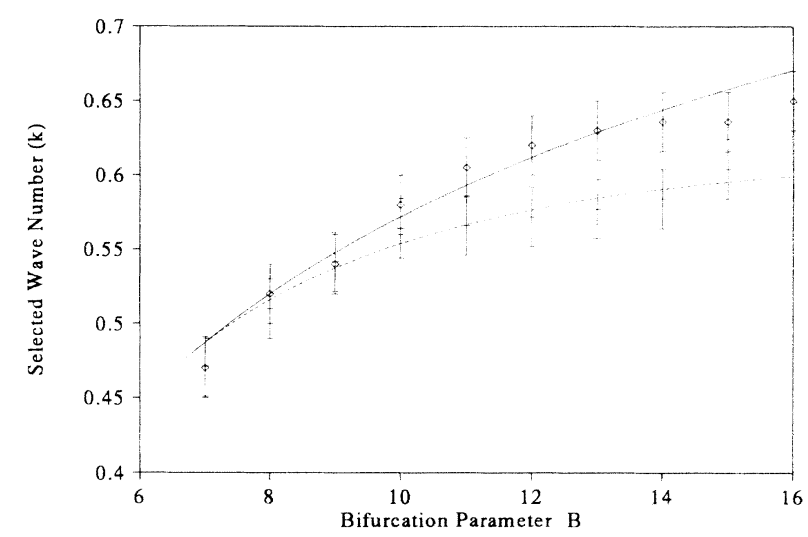

FIG. 8. Wave number selection for the Brusselator model. The fully drawn curve shows the wave number for the modes of maximum linear gain as a function of $B$. The dotted curve represents the analytical results for the wave number selected behind a traveling front. Diamonds and crosses are numerically determined results for the final stationary patterns developed from the homogeneous steady state and behind a traveling front, respectively.

serve (i) that the finally selected large signal wave numbers generally agree with those predicted by the small signal theory, (ii) that the two types of selection processes lead to similar wave numbers close to the bifurcation point, and (iii) that far from the bifurcation point largerwavelength structures are produced behind a traveling front than from the homogeneous steady state. For large $B$ values there is a tendency for the stationary wave numbers developed from the homogeneous steady state to fall below the wave numbers of maximum linear gain and to approach those developed behind a front. This far from the bifurcation point the growth rates of the unstable modes are so high that it is difficult to stabilize a homogeneously growing structure. Instead, patches will develop, and as they spread into neighboring regions of space, patterns with smaller wave numbers will be produced.

Figure 9 shows the variation of the front velocity with $B$ for the one-dimensional Brusselator model. The fully drawn curve represents the results of the above analytical approach, and the points with error bars show the numerically obtained results for the fully developed structure. In this case, the Turing bifurcation is supercritical, and the front velocity goes to zero in the bifurcation point $(B \cong 6.7$ ).

We may conclude this discussion by noticing that in a region just above the bifurcation point $b_{T}<b<p_{\text {low }}$, the Turing structure is stronger than the homogeneous steady state. Hence, if a system is prepared such that both states are present, the Turing structure will gradually spread across the entire system. In the interval $p_{\text {low }}<b<p_{\text {high }}$, however, the two states can coexist in a stable manner in different regions of the system. As described in more detail by Jensen et al. [31], this pinning phenomenon allows one to obtain an infinite variety of stable heterogeneous structures in which, for instance, an islet of Turing stripes exists in a sea of homogeneous steady state. 


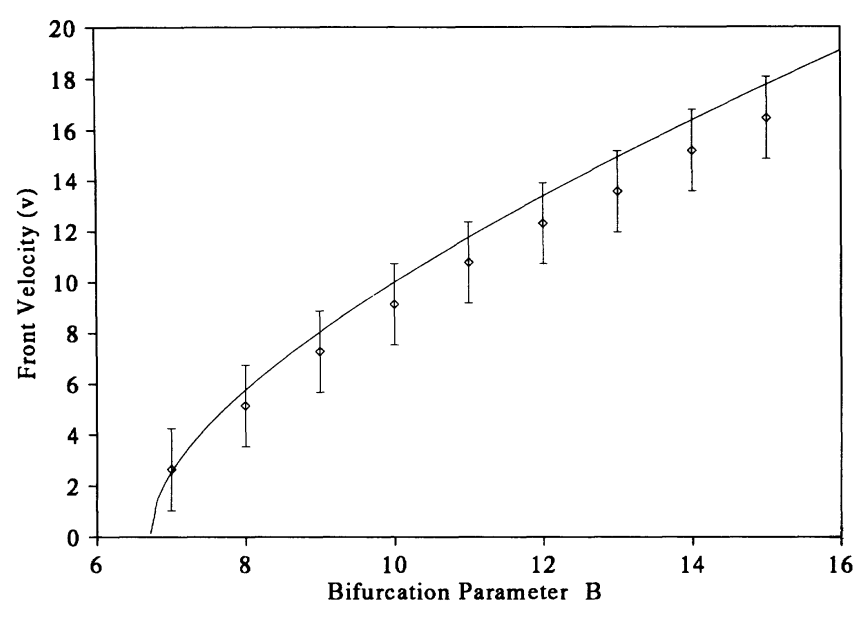

FIG. 9. Variation of the front velocity for a one-dimensional Brusselator model. The fully drawn curve represents the analytical results, and the diamonds with error bars represent numerically obtained results. The corresponding bifurcation diagram shows that the Turing bifurcation in this case is supercritical.

The possibility that an islet of one phase can be stably embedded in a sea of another arises from the fact that we are dealing with a nonequilibrium phase transition. In this case there is no general principle that predicts the existence of a single stable phase. If one phase involves a modulation in space, a finite amount of disequilibrium between the two phases is needed to make the interface move $[37,38]$, and pinning of the interface to the structure can occur in parameter regions where only a small disequilibrium exists. A more detailed analysis of this phenomenon has been given by Bensimon, Shraiman, and Croquette [39]. They consider a model that describes a subcritical bifurcation between conductive and convective states in a 1D hydrodynamic system and show how the front can be pinned by the roll structure of the convective state. There are important differences between the two models, however, which among other things lead to the fact that where the front propagation in our system occurs in jumps of one wavelength of the underlying structure, the analysis of the hydrodynamic system leads to jumps of a half-wavelength.

\section{NUCLEATION AND GROWTH OF 2D HEXAGONAL STRUCTURES}

In 2D the first structure to appear subcritically has hexagonal symmetry where the concentration maxima for the activator form a triangular lattice. This is followed on decreasing $b$ and also subcritically by stripes. In a certain parameter range the system may thus exhibit tristability between hexagons, stripes and the homogeneous steady state. At still lower values for $b$ (and somewhat higher values for $\delta$ ), a branch of inverted hexagons exists for which the activator concentration is minimum in the hexagonal lattice points [40]. Pinning may occur whenever the boundary of the structure is perpendicular (or nearly perpendicular) to the wave vectors characterizing the pattern [41]. As a result, a large variety of localized 2D structures are possible. Outside the pinning band, localized structures may either shrink or expand, depending on the relative strength of the surrounding state. However, the way this process takes place is still influenced by pinning phenomena and is qualitatively different from the growth of localized hexagonal structures in models, for instance, with Brusselator-type kinetics. As an example, Fig. 10 shows the growth of a hexagonal pattern embedded in a sea of homogeneous steady state. To produce this figure, we have initiated the system with seven spots of a perfect hexagonal pattern. With a bifurcation parameter $b=2.5$ we are well above the pinning band, and the Turing structure grows with time as illustrated by the sequence in Fig. 10. It is interesting to see how this growth occurs through the addition of new hexagonal points in a systematic manner.

A hexagonal pattern can be considered as the superposition of three stripe patterns with wave vectors $120^{\circ}$ apart. As illustrated in Fig. 11, the pinning directions, i.e., the directions in which expansion of the structure meets with the highest resistance, are parallel to these wave vectors. Whether a new spot will start to form also depends on the number of surrounding spots that are already developed. Many developed spots in the neighboring lattice points will provoke growth. Since pinning is strongest in directions $A$, new hexagonal spots will first develop along the $B$ directions (see Fig. 11). Hereafter, pinning can no longer hold back nucleation of new spots in the $A$ directions because of the high number of developed spots on the neighboring lattice sites. Hence, the overall hexagonal form of the structure is reestablished, and growth again occurs in the $B$ directions, etc.

In this way it appears that pinning effects related to the interacting stripe patterns influence the growth of the hexagonal structure. When the bifurcation to stripes is supercritical, there is no pinning of the patterns, and the hexagonal structure expands in a qualitatively different manner. This is illustrated in Fig. 12 for the Brusselator model. Here we see how stripes are formed along each of the hexagonal sides of the structure, and how these stripes subsequently break up into hexagonal spots. This type of "crystalization" has also been observed in experiments with convection cells in gaseous $\mathrm{CO}_{2}$ [42]. The bifurcation diagram for this system (Fig. 3 of Ref. [39]) shows that just above the bifurcation point a branch of metastable stripes coexists with a branch of stable hexagons. This provides a likely explanation for the observed formation of transient stripe patterns. It may be noted that around the Turing point, the bifurcation diagram for the Brusselator model [28] is qualitatively similar to that of the convection problem.

The Lengyel-Epstein model also allows for the existence of stable, localized 2D structures: hexagonal patterns in a sea of homogeneous steady state, hexagonal patterns surrounded by a state of stripes mixed with hexagons, and - under very particular circumstances-a region with self-sustained oscillations surrounded by a ring structure of stripes. As an example, Fig. 13 shows a stable, localized hexagonal structure obtained for $b=3.6$. To check the stability of this structure, noise has been 


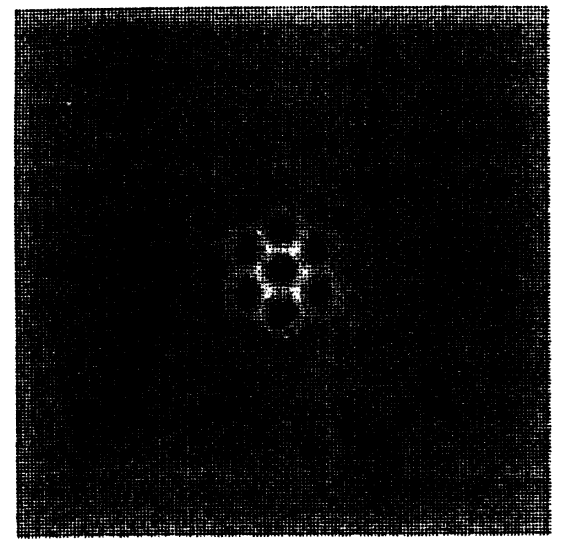

Time $=0.047407$

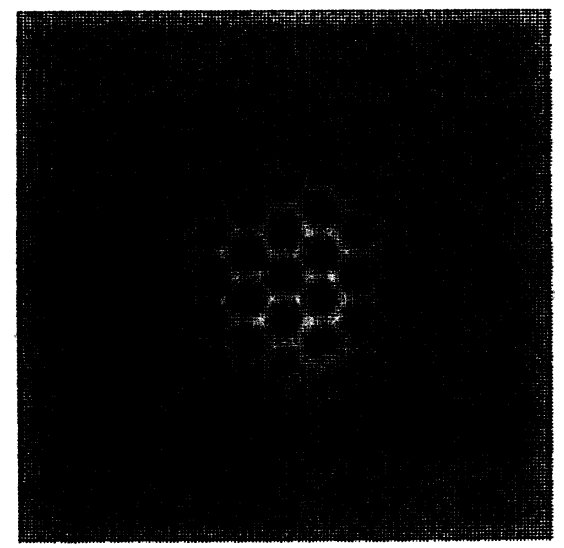

Time $=5.736296$

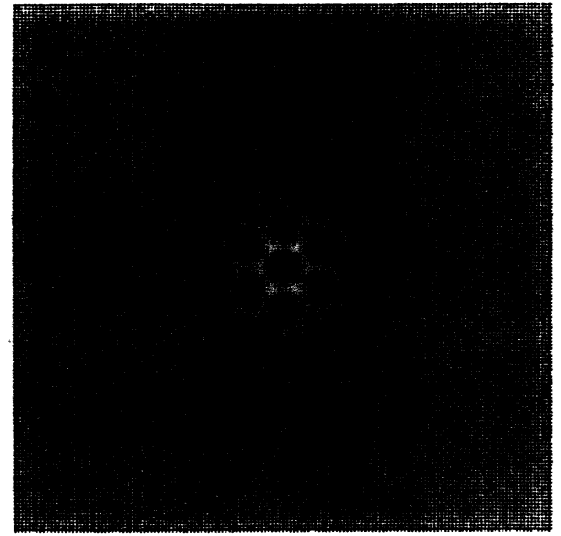

Time $=1.943704$

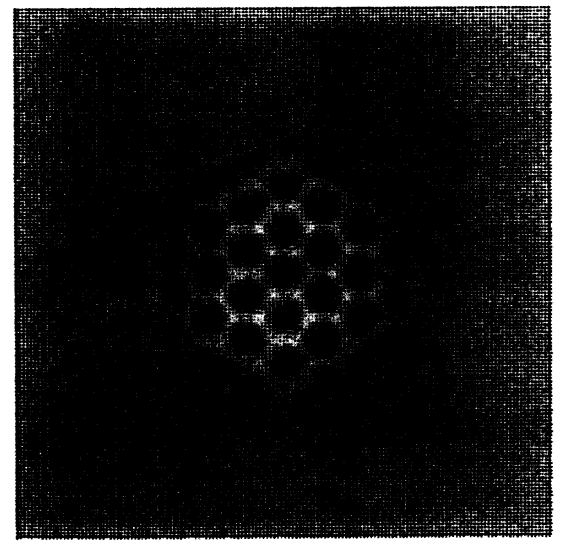

Time $=7.632593$

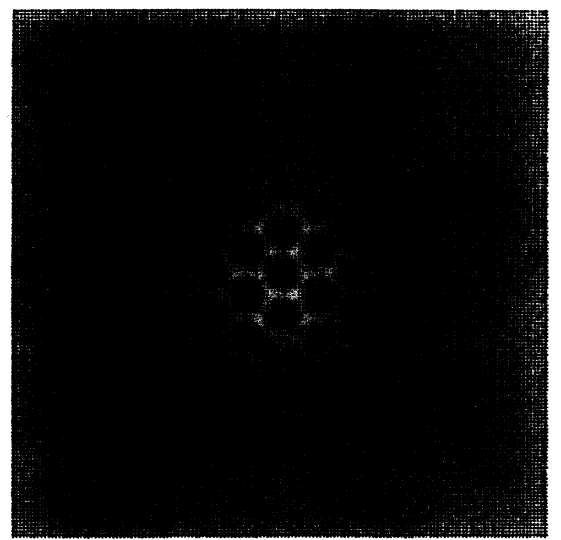

Time $=3.840000$

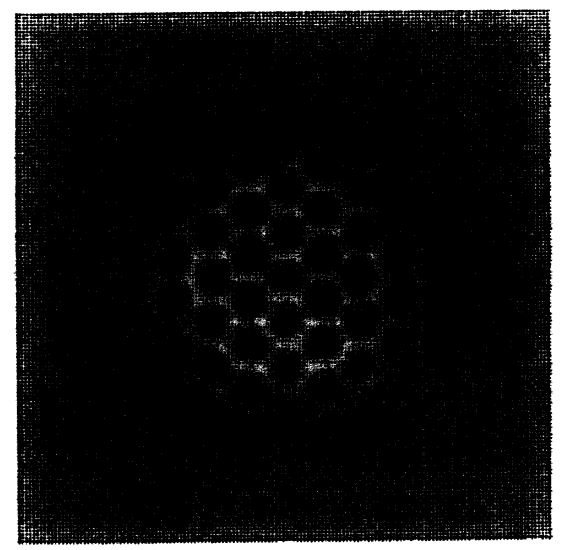

Time $=9.528889$

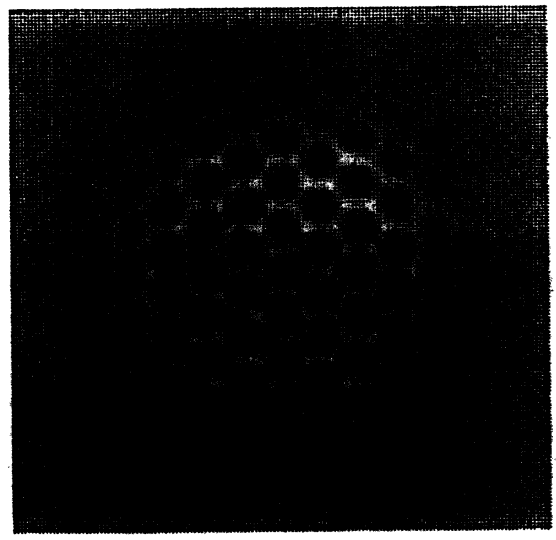

Time $=13.321481$

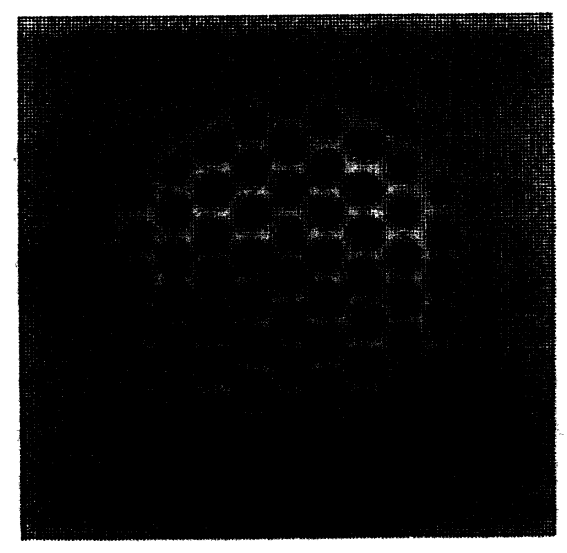

Time $=15.217778$

Time $=11.425185$

FIG. 10. Expanding hexagonal pattern in the Lengyel-Epstein model with $b=2.5$. Note how new hexagonal points are added in directions where pinning can be overcome. 

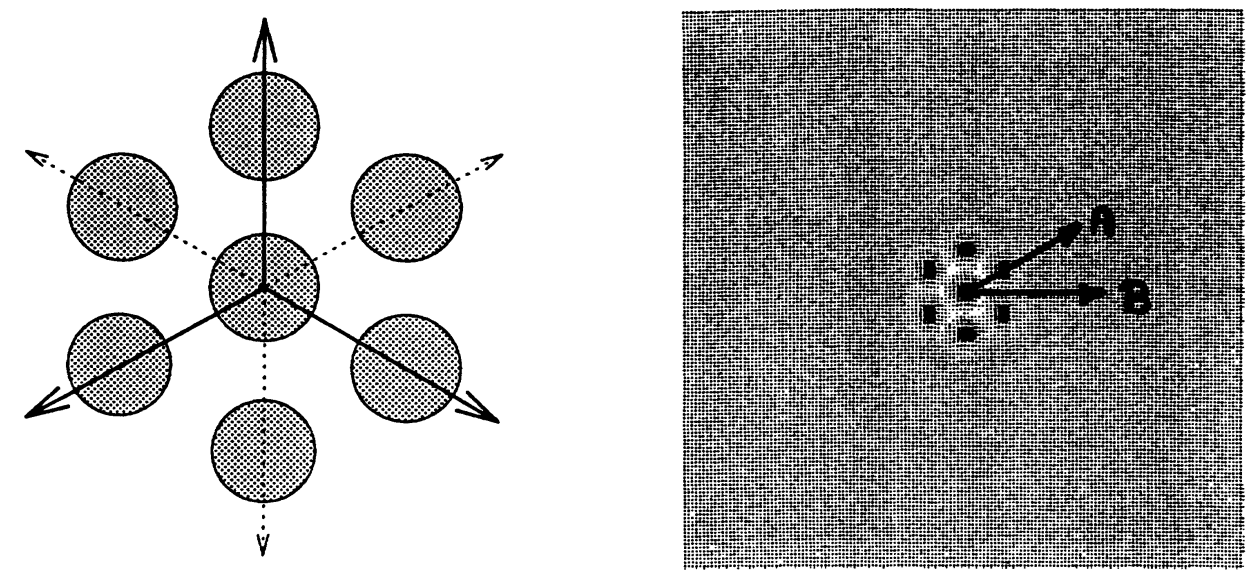

FIG. 11. The three waves vectors characterizing a hexagonal structure. $A$ is the pinning direction.

added during the simulation, and the simulation has been continued for an extended period of time. If $b$ is further increased, the homogeneous steady state becomes stronger than the hexagonal structure, and the Turing structure will shrink. We have also seen examples of numerical simulations in which the Turing pattern starts to decay into the homogeneous steady state at points inside the structure.

\section{1D AND 2D SPIRALS. TURBULENCE}

In the region of parameter space where Turing stripes and Hopf oscillations are both stable (and where the mixed Turing-Hopf mode is unstable), fronts connecting these two global solutions exist [32]. Close to the Hopf bifurcation, the Turing state is dominant and tends to invade the territory of the Hopf oscillations. Near the lower end of the coexistence range, the opposite occurs. In these processes, the front velocity exhibits characteristic jumps as previously discussed for a front between Turing stripes and the homogeneous steady state. Only in this case, one wavelength is usually added to (or subtracted from) the Turing domain during an integer periods of the Hopf oscillation. Hence, we have a phenomenon analogous to the well-known frequency locking between coupled nonlinear oscillators. It is likely that quasiperiodic and perhaps chaotic motions of the front also can occur. These problems are presently being investigated in more detail.

Because of pinning effects, stationary fronts between a Turing and a Hopf domain can arise in certain parameter regions. Such fronts may again serve as building blocks for stable localized structures such as, for instance, a Turing structure embedded in a sea of Hopf oscillations. An example is shown in Fig. 14, where a 1D stripe structure truncated to a few wavelengths is surrounded on both sides by Hopf oscillations. The amplitude of the oscillations goes to zero in the core of the structure where the Turing amplitude is maximum. The Turing fronts serve as sources of waves that travel to either side. For a nar- row Turing structure consisting of one or a few halfwavelengths, the waves are released with opposite phases (corresponding to a phase jump of $\pi$ ). In the case of a broader Turing structure, the interaction between the confining fronts is less significant, and the phase difference between the initiated waves may differ from $\pi$. Due to a subtle compensation between the spatial and nonlinear dispersion effects, the wave number for these waves is very small, and the phase velocity correspondingly high. Similar asynchronous wave sources (so-called chemical flip-flops) have been observed in experiments with the CIMA reaction [21]. The phenomenon has also been found in numerical simulations of the Brusselator model [22]. Only in this case, the phase velocity of the emitted waves is more moderate.

To obtain the results of Fig. 14 we have initiated the right half-plane and the left half-plane in stable Hopf oscillations with a phase difference of $\pi$ between the two sides. Hereafter, we have simulated the Lengyel-Epstein model until all transient behaviors have died out.

In $2 \mathrm{D}$, a $2 \pi$ phase singularity can be imposed onto the system by starting each quadrant of the plane in a stable Hopf oscillation with a phase difference of $\pi / 2$ from quadrant to quadrant around the clock. For conditions similar to those of Fig. 14 this produces a stable 2D spiral with a Turing induced core as illustrated in Fig. 15. The arms of the spiral are rather straight, expressing the aforementioned high phase velocity of the emitted waves. This type of 2D Turing-Hopf spiral has recently been observed by De Kepper et al. [43].

By virtue of the built-in Turing core, the spirals of Figs. 14 and 15 are quite different from the well-known chemical spirals observed, for instance, in the BelousovZhabotinsky reaction [4,5]. However, in the absence of starch (i.e., for $\delta=1$ ), one can find 2D spirals without Turing cores in the Lengyel-Epstein model as well. We have only just initiated work in this direction. It is clear, however, that for certain parameter values, the spirals are destabilized and for large systems, defect mediated turbulence may arise. This phenomenon is characterized by the continuous creation and annihilation of phase singu- 


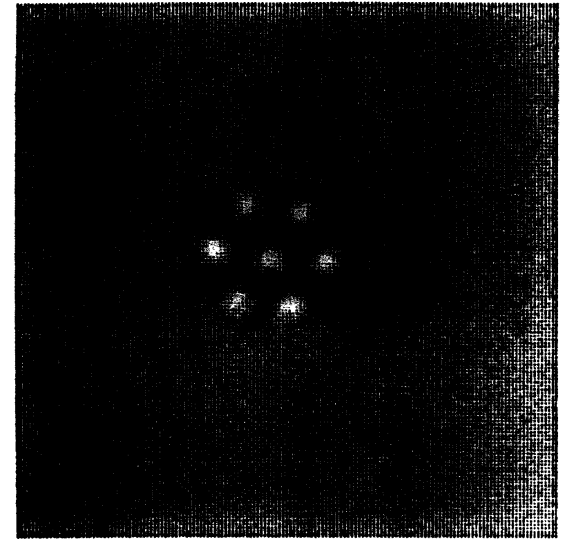

Time $=0.035714$

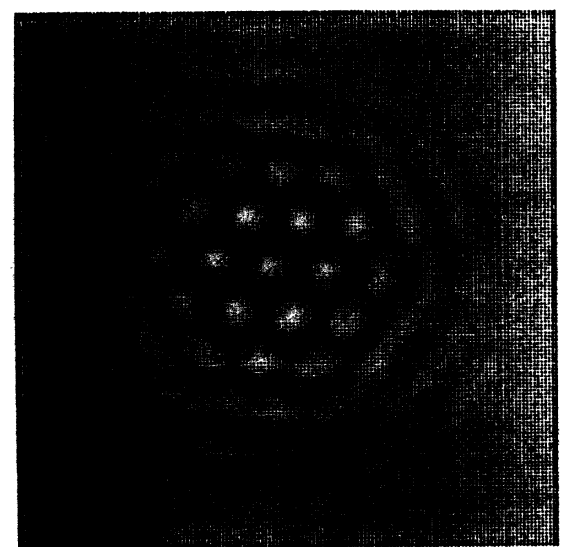

Time $=30.025714$

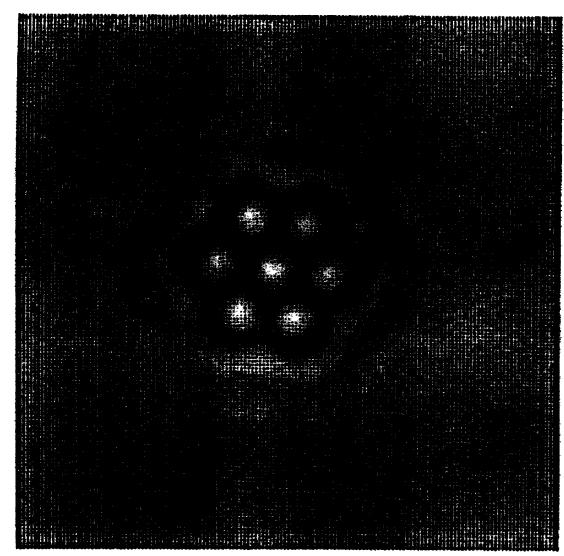

Time $=10035714$

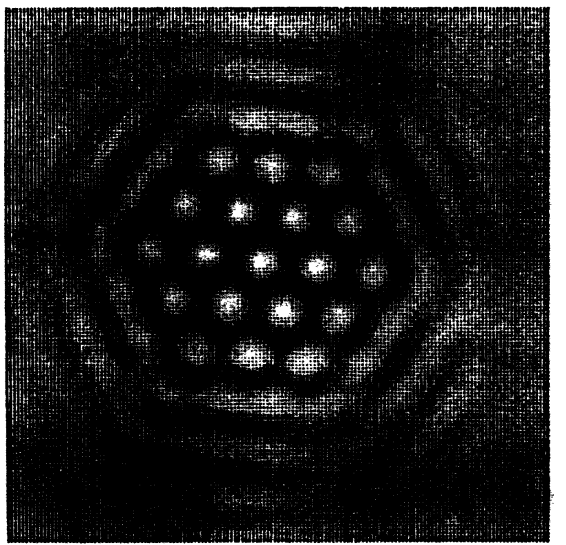

Time $=40.035714$

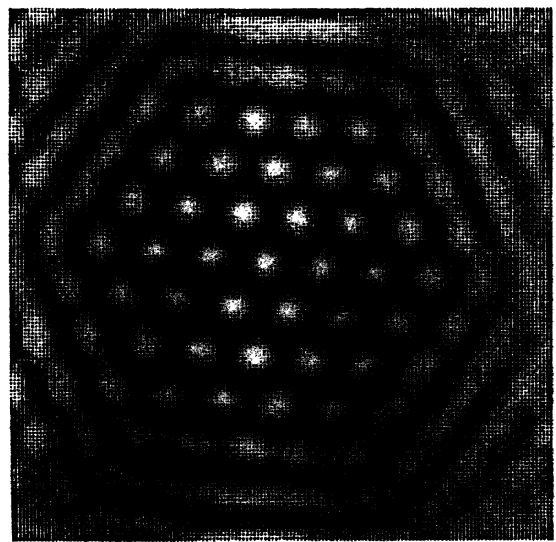

Time $=70035714$

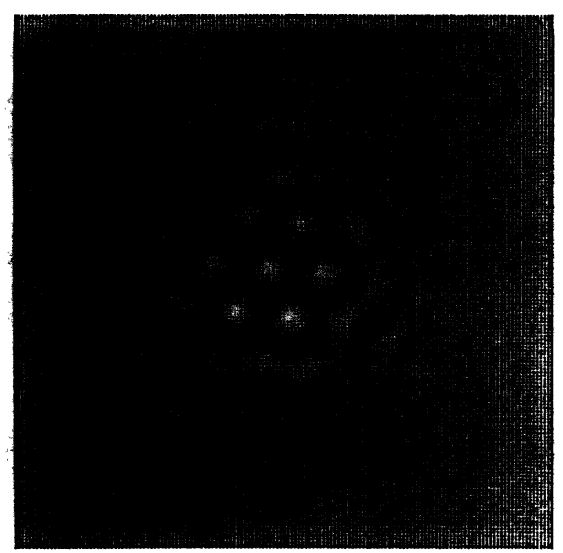

Time $=200357: 4$

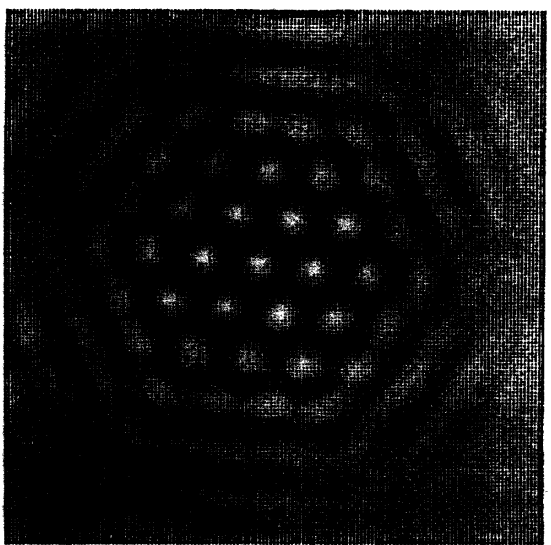

Time $=50035714$

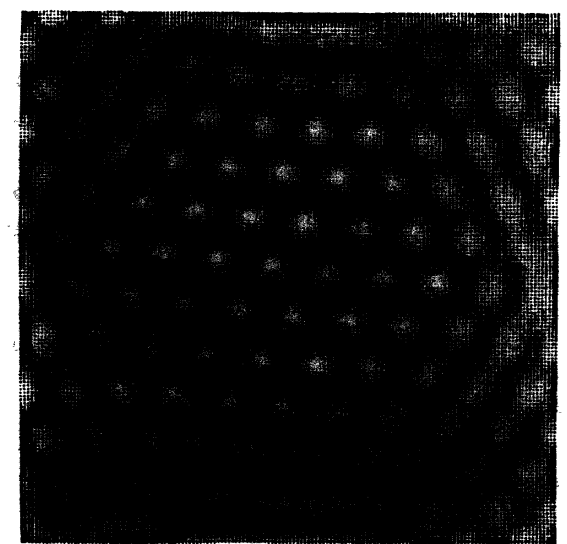

Time $=80.035714$

Time $=60.035714$

FIG. 12. Expanding hexagonal pattern in the Brusselator model with $A=4.5, B=6.8, D_{x}=7$, and $D_{y}=56$. Note how this expansion occurs in a way that is qualitatively different from that of Fig. 10. 


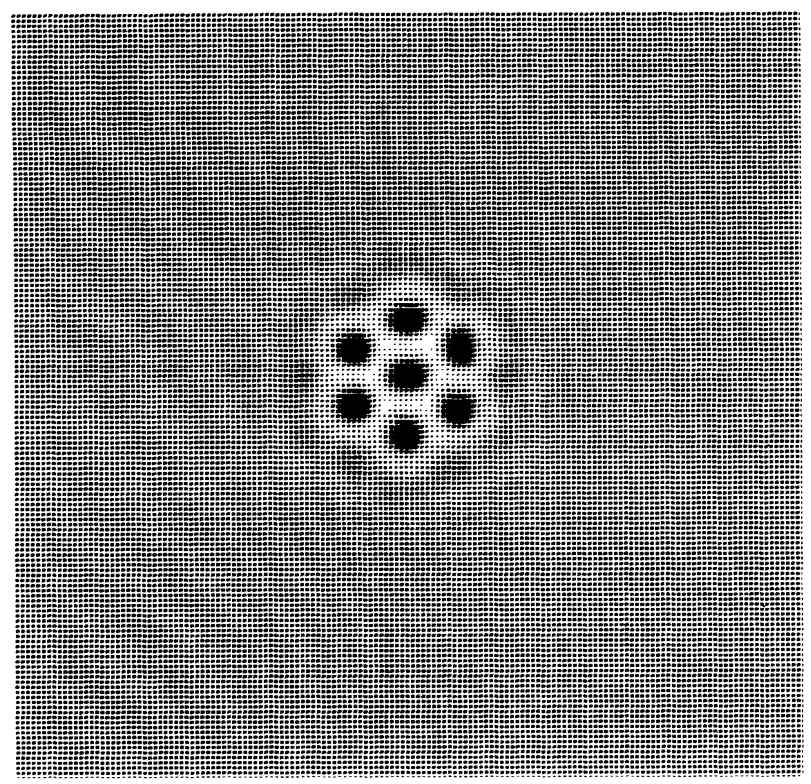

FIG. 13. Localized hexagonal structure in the LengyelEpstein model for $b=3.6$. larities out of a weak externally imposed random noise signal. The turbulent state is also characterized by the apparently random motion of the phase singularities between one another. For other parameters, after a longer or shorter transient, the system may settle down into a frozen state with a network of interlocking phase singularities.

\section{CONCLUSIONS}

The Lengyel-Epstein model is presumably the best available representation of the mechanisms believed to be responsible for the recently observed Turing structures in the so-called CIMA reaction. In the absence of specific symmetries, as is usually the case for chemical reactiondiffusion systems, the transition from the homogeneous steady state to hexagons is subcritical $[44,45]$. Numerically calculated bifurcation diagrams for this model show that the Turing bifurcation to stripes is strongly subcritical. This makes the model qualitatively different from previously studied, simple reaction-diffusion models capable of producing Turing structures, hence warranting a more detailed study of structure formation and front

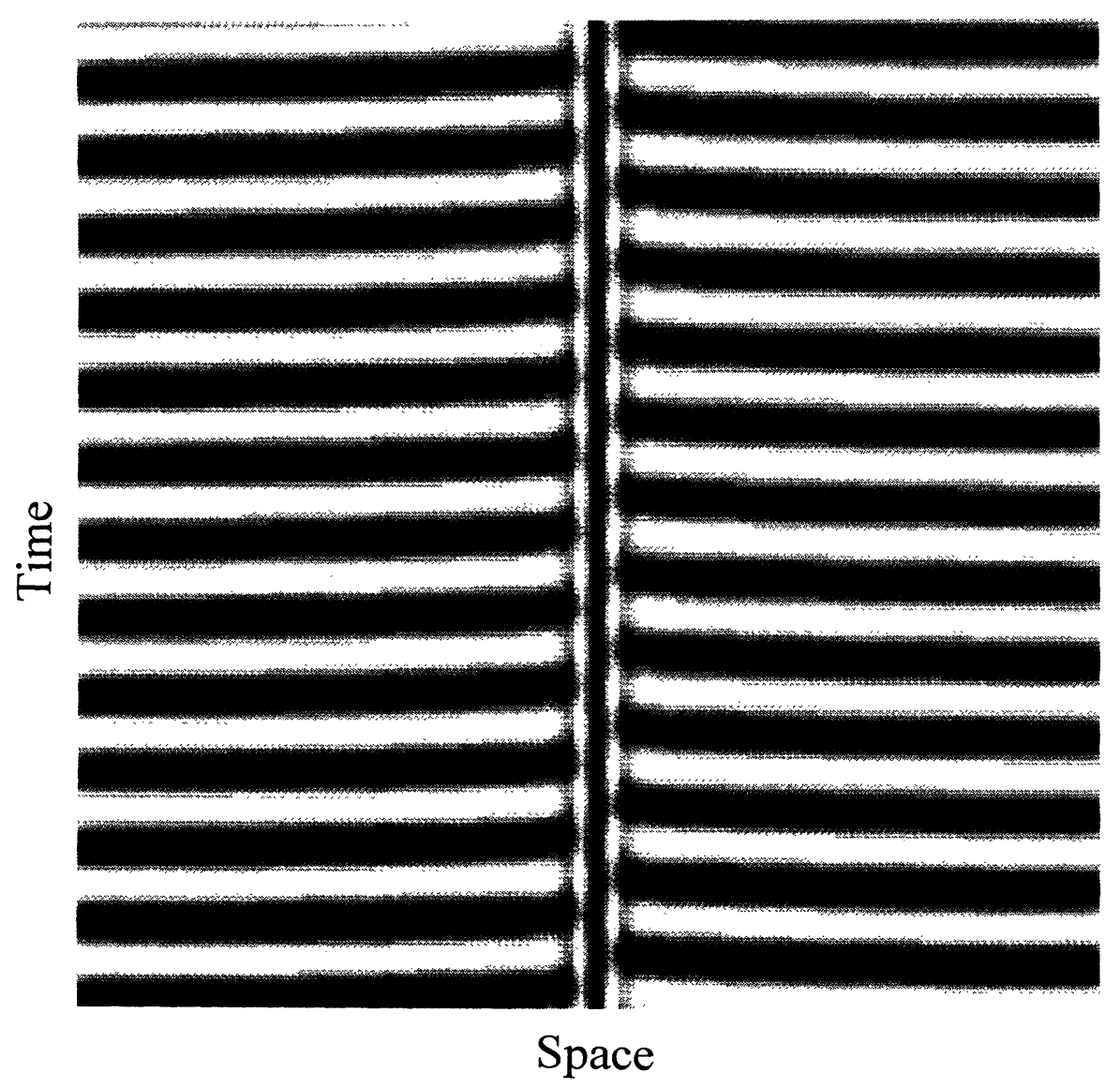

FIG. 14. Chemical flip-flop or 1D spiral in the Lengyel-Epstein model for $a=30, b=1.1$, $c=1.5$, and $\delta=8$. A $1 \mathrm{D}$ stripe structure is embedded in a background of nearly homogeneous oscillations. 


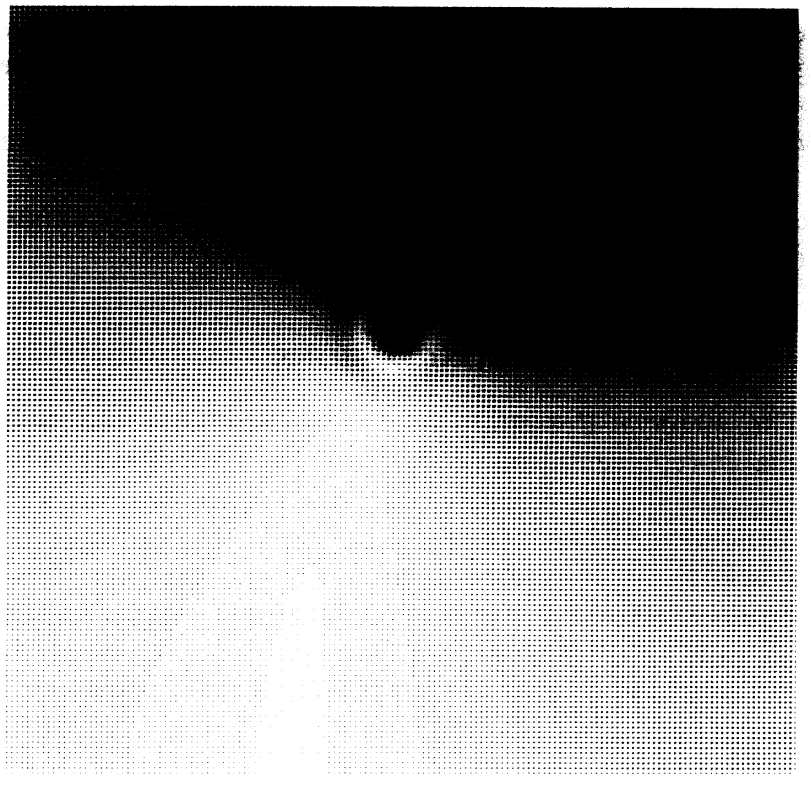

FIG. 15. 2D spiral with a Turing-induced core. Parameters are the same as Fig. 14. The rotation of the spiral is clockwise. For symmetry reasons, a similar spiral rotating in the opposite direction must also exist.

propagation in this model. In particular, the relatively pronounced character of the subcriticality allows for the existence of a pinning band in which a wide variety of stable inhomogeneous structures can arise.

In the present paper we have derived simple analytical results for the velocity of a front between Turing stripes and the homogeneous steady state as well as for the wave number selected behind the front. Due to the lineariza- tion involved, these results apply only in the supercritical region well away from the bifurcation point. Numerically results are presented to complement the analytical results outside this range.

In the two-dimensional case we have studied the growth of droplets of hexagonal structure into the surrounding homogeneous steady state. These results differ from those observed for other simple reaction-diffusion models by the absence of stripes in front of the growing hexagonal structure. Similar investigations in the region where competition exists between Turing structures and Hopf oscillations provide evidence of pinning effects both in one and in two dimensions [32].

Using appropriate initial conditions, we have found that one- and two-dimensional spirals can develop in the oscillatory domain. These spirals have a Turing-induced core where the amplitude of the oscillations vanishes. In one dimension, the oscillations have opposite phases on either side of the core similar to the quasi-onedimensional spirals observed in the experiments with the CIMA reaction [21]. With the right set of parameters, starting in the homogeneous steady state the system can end up in a frozen state with a finite number of fixed phase singularities. In the inverted region of the Hopf bifurcation we have also found turbulent states. Work is presently underway to investigate these phenomena in greater detail.

\section{ACKNOWLEDGMENTS}

We would like to thank Professor G. Nicolis for his interest in this work. Discussions with P. De Kepper, A. De Wit, and S. Metens are acknowledged. The work was supported by the EC Erasmus Program B 1028/13 and sponsored by Twinning Contract No. SC1 ${ }^{*}$-CT91-0706 of the EC Science Program and by FNRS (Belgium).
[1] A. M. Turing, Philos. Trans. R. Soc. London Ser. B 327, 37 (1952).

[2] G. Nicolis and I. Prigogine, Self-Organization in Nonequilibrium Systems (Wiley, New York, 1977).

[3] H. Haken, Synergetics, An Introduction (Springer-Verlag, Berlin, 1977).

[4] Y. Kuramoto, Chemical Oscillations, Waves and Turbulence (Springer-Verlag, Berlin, 1984).

[5] Oscillations and Traveling Waves in Chemical Systems, edited by D. Field and M. Burger (Wiley, New York, 1985).

[6] H. Meinhardt, Models of Biological Pattern Formation (Academic, London, 1982).

[7] J. D. Murray, Mathematical Biology (Springer-Verlag, Berlin, 1989).

[8] Complexity Chaos and Biological Evolution, Vol. 270 of NATO Advanced Study Institute, Series B: Physics, edited by E. Mosekilde and Li. Mosekilde (Plenum Press, New York, 1990).

[9] Hydrodynamic Instabilities and Transition to Turbulence, 2nd ed., edited by H. L. Swinney and J. P. Gollub (Springer-Verlag, Berlin, 1985).
[10] P. Manneville, Dissipative Structures and Weak Turbulence (Academic, Boston, 1990).

[11] Nonlinear Evolution of Spatio-Temporal Structures in Dissipative Continuous Systems, Vol. 255 of NATO Advanced Study Institute, Series B: Physics, edited by F. H. Busse and L. Kramer (Plenum, New York, 1990).

[12] A. C. Newell and J. V. Moloney, Nonlinear Optics (Addison-Wesley, Redwood City, CA, 1992).

[13] K. Shoichi, S. C. Müller, and J. Ross, J. Chem. Phys. 76, 1392 (1982).

[14] S. C. Müller, T. Plesser, and B. Hess, Science 230, 661 (1985).

[15] Z. Noszticzius, W. Horsthemke, W. D. McCormick, and H. L. Swinney, Nature 329, 619 (1987).

[16] R. D. Vigil, Q. Ouyang, and H. L. Swinney, Physica A 188, 17 (1992).

[17] V. Castets, E. Dulos, J. Boissonade, and P. De Kepper, Phys. Rev. Lett. 64, 2953 (1990).

[18] Q. Ouyang and H.L. Swinney, Nature (London) 352, 610 (1991).

[19] I. Lengyel and I. R. Epstein, Proc. Natl. Acad. Sci. U.S.A. 89, 3977 (1992). 
[20] A. Hunding and P. G. Sørensen, J. Math. Biol. 26, 27 (1988).

[21] J. J. Perraud, K. Agladze, E. Dulos, and P. De Kepper, Physica A 188, 1 (1992).

[22] J. J. Perraud, A. De Wit, E. Dulos, P. De Kepper, G. Dewel, and P. Borckmans, Phys. Rev. Lett. 71, 1272 (1993).

[23] I. Lengyel, G. Rábai, and I. R. Epstein, J. Am. Chem. Soc. 112, 4606 (1990).

[24] I. Lengyel, G. Rábai, and I. R. Epstein, J. Am. Chem. Soc. 112, 9104 (1990).

[25] I. Lengyel and I. R. Epstein, Science 251, 650 (1991).

[26] A. De Wit, G. Dewel, P. Borckmans, and D. Walgraef, Physica D 61, 289 (1992).

[27] P. Borckmans, A. De Wit, and G. Dewel, Physica A 188, 137 (1992).

[28] J. Verdasca, A. De Wit, G. Dewel, and P. Borckmans, Phys. Lett. A 168, 194 (1992).

[29] V. Dufiet and J. Boissonade, J. Chem. Phys. 96, 664 (1992).

[30] V. Dufiet and J. Boissonade, Physica A 188, 158 (1992).

[31] O. Jensen, V. O. Pannbacker, G. Dewel, and P. Borckmans, Phys. Lett. A 179, 91 (1993).

[32] V. O. Pannbacker, O. Jensen, G. Dewel, P. Borckmans, and E. Mosekilde, in Spatio-Temporal Patterns in Nonequilibrium Systems, edited by P. Palffy-Muhoray and P. E.
Cladis (Kluwer, Amsterdam, in press).

[33] E. Ben-Jacob, H. Brand, G. Dee, L. Kramer, and J. S. Langer, Physica D 14, 348 (1985).

[34] W. van Saarloos, Phys. Rev. A 37, 211 (1988).

[35] W. van Saarloos, Phys. Rev. A 39, 6367 (1989).

[36] W. van Saarloos and P. C. Hohenberg, Phys. Rev. Lett. 64, 749 (1990).

[37] Y. Pomeau, Physica D 23, 3 (1986).

[38] O. Thual and S. Fauve, J. Phys. (Paris) 49, 1829 (1988).

[39] D. Bensimon, B. J. Shraiman, and V. Croquette, Phys. Rev. A 38, 5461 (1988).

[40] V. O. Pannbacker, O. Jensen, E. Mosekilde, G. Dewel, and P. Borckmans, in Proceedings of the 1st Copenhagen Symposium on Computer Simulation in Biology, Ecology and Medicine, edited by E. Mosekilde (Simulation Councils, San Diego, 1993), p. 103.

[41] B. A. Malomed, A. A. Nepomnyashchii, and M. J. Tribelskii, Phys. Rev. A 42, 7244 (1990).

[42] E. Bodenschatz, J. R. de Bruyn, G. Ahlers, and D. S. Cannell, Phys. Rev. Lett. 67, 3078 (1991).

[43] P. De Kepper, J. J. Perraud, B. Rudovics, and E. Dulos, J. Bifurc. Chaos (to be published).

[44] F. H. Busse, J. Fluid Mech. 30, 625 (1967).

[45] L. M. Pismen, J. Chem. Phys. 72, 1900 (1980). 


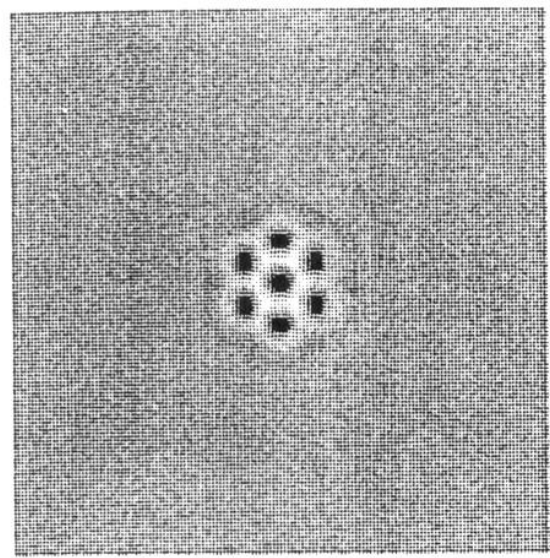

Time $=0.047407$

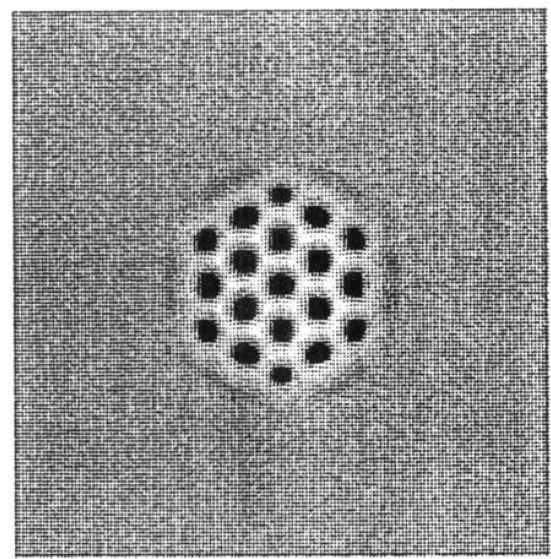

Time $=5.736296$

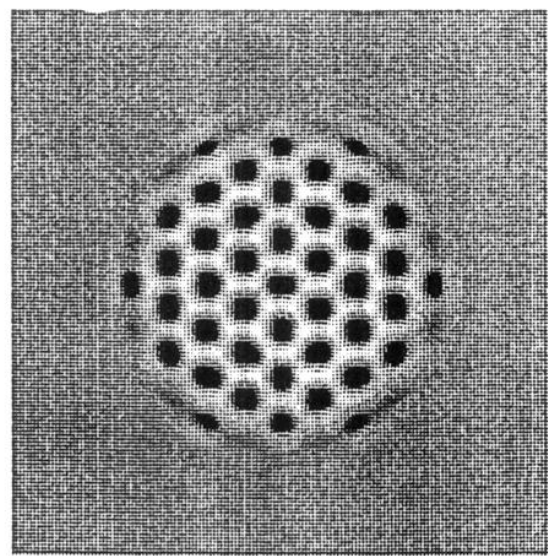

Time $=11.425185$

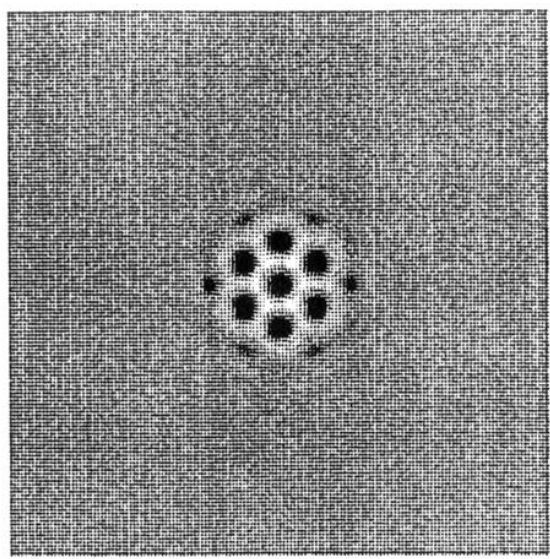

Time $=1.943704$

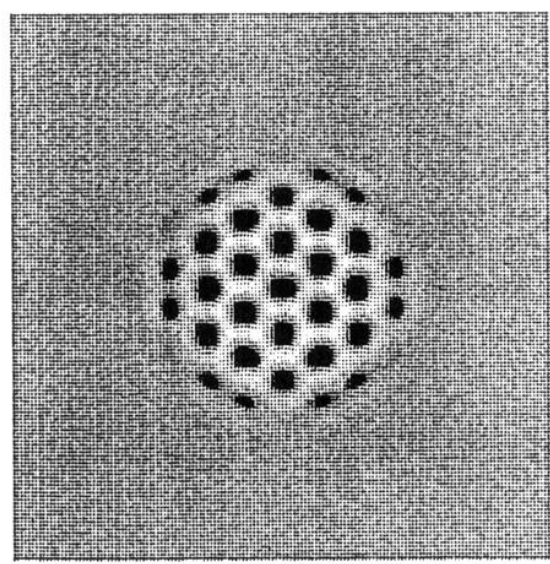

Time $=7.632593$

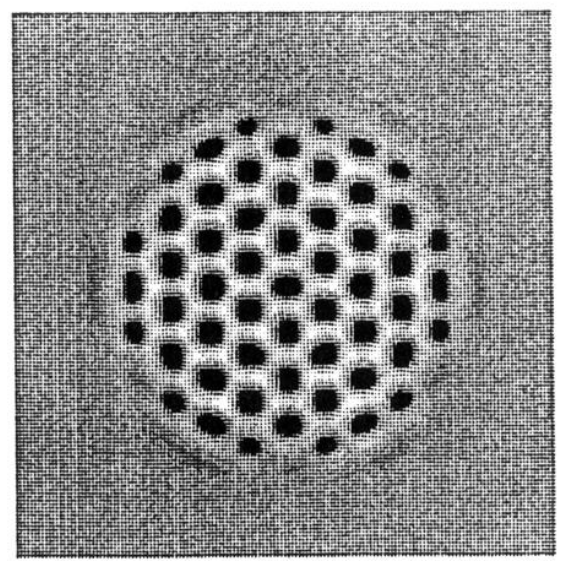

Time $=13.321481$

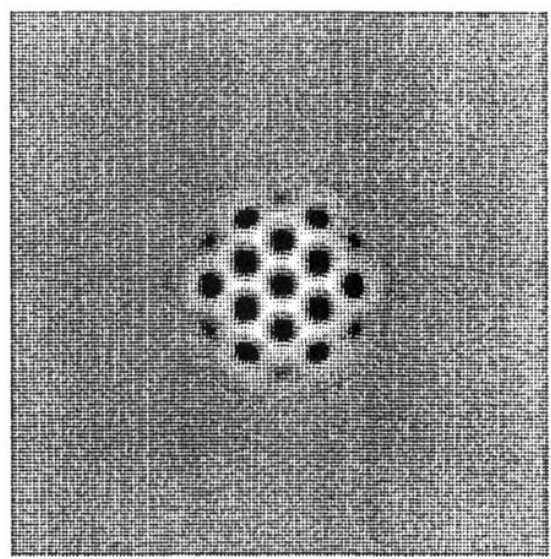

Time $=3.840000$

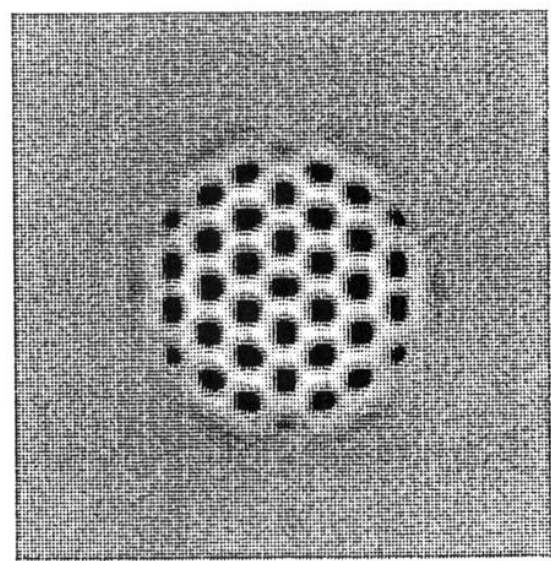

Time $=9.528889$

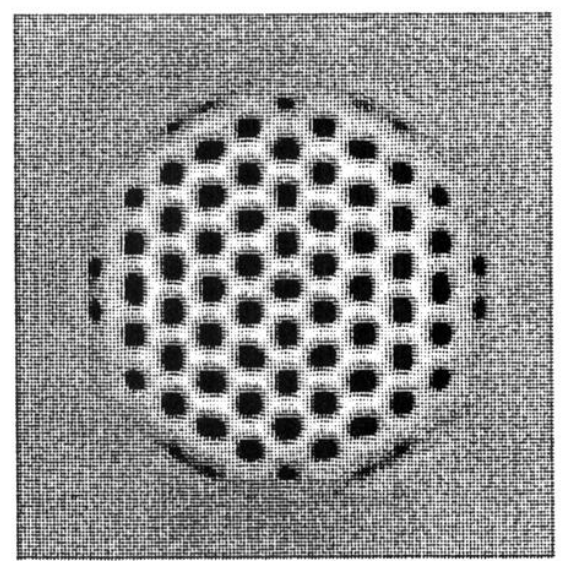

Time $=15.217778$

FIG. 10. Expanding hexagonal pattern in the Lengyel-Epstein model with $b=2.5$. Note how new hexagonal points are added in directions where pinning can be overcome. 

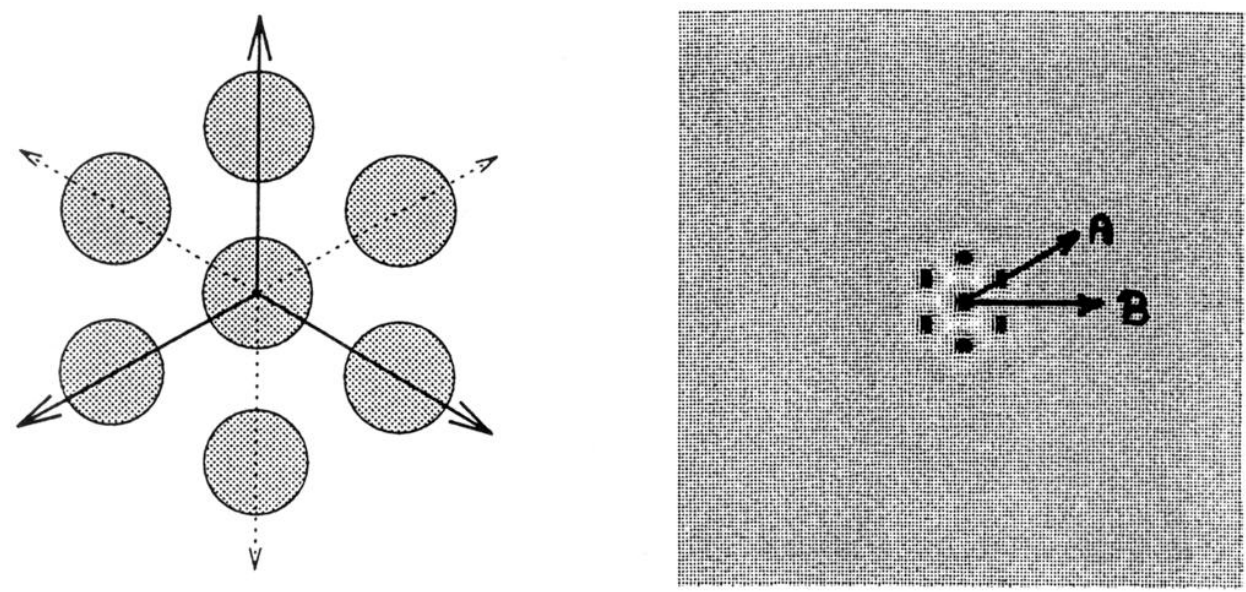

FIG. 11. The three waves vectors characterizing a hexagonal structure. $A$ is the pinning direction. 


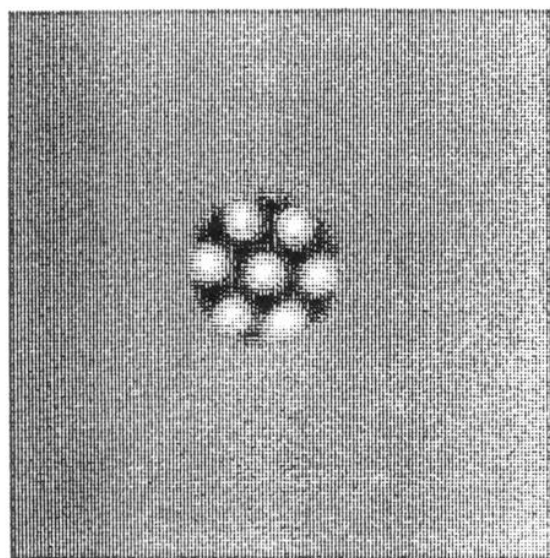

Time $=0.035714$

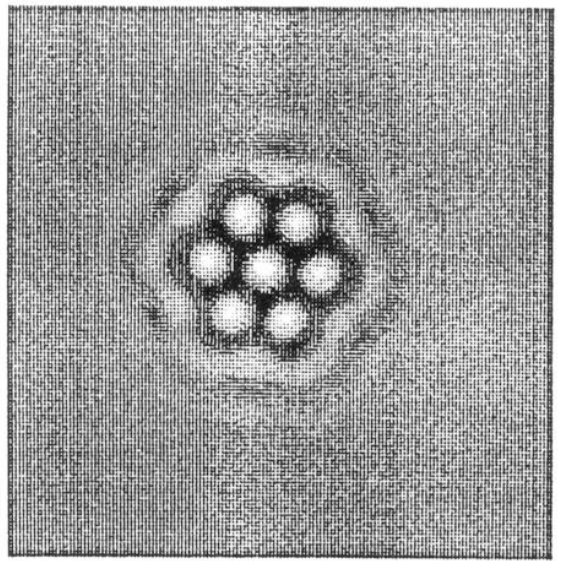

Time $=10.035714$

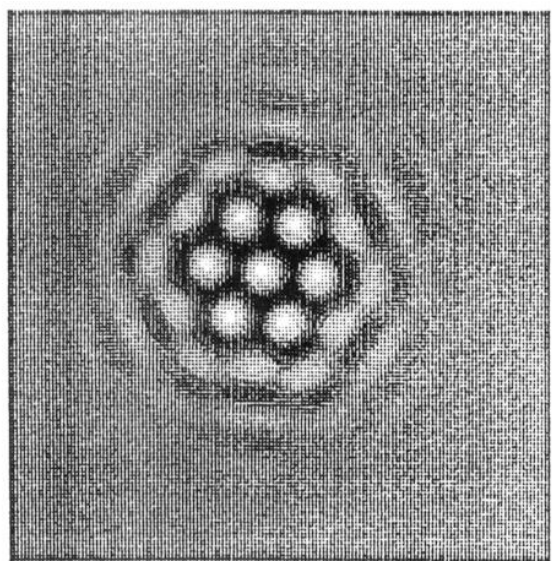

Time $=20.035714$

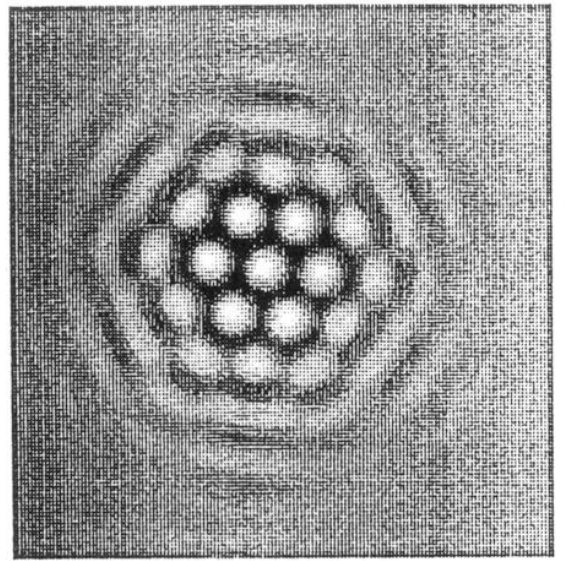

Time $=30.035714$

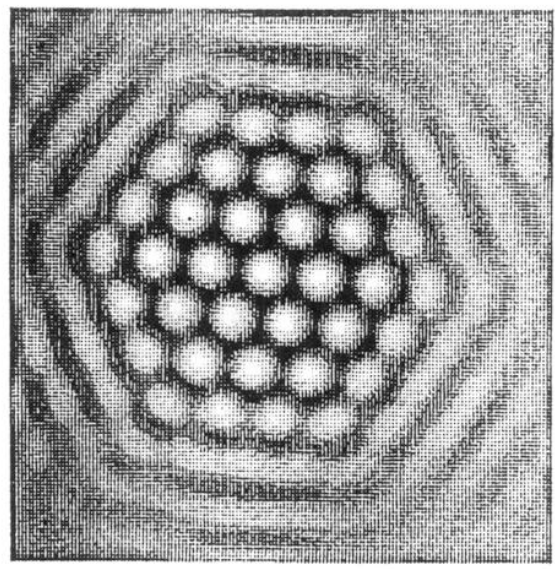

Time $=60.03571 \mathrm{~A}$

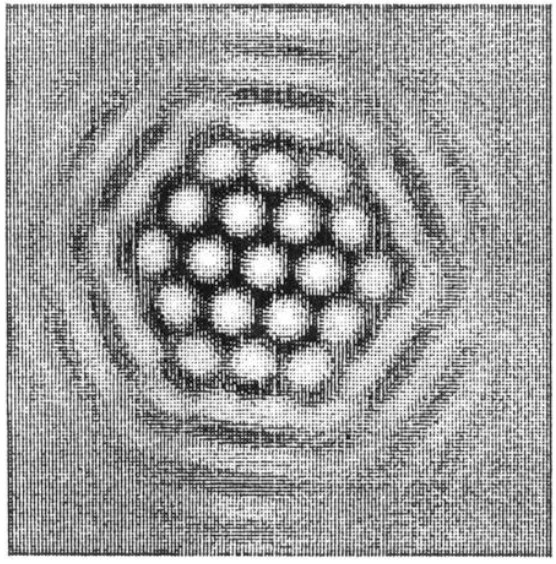

Time $=40.035714$

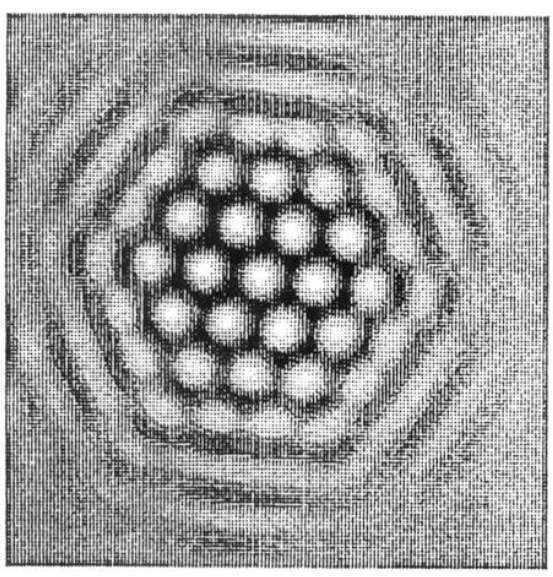

Time $=50.035714$

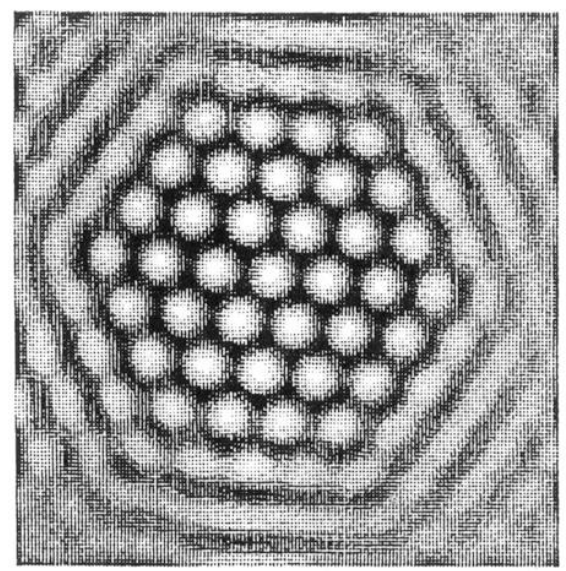

Time $=70.035714$

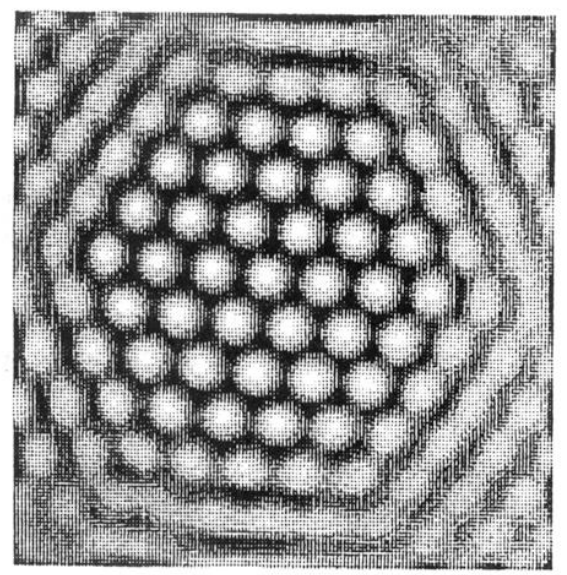

Time $=80.035714$

FIG. 12. Expanding hexagonal pattern in the Brusselator model with $A=4.5, B=6.8, D_{x}=7$, and $D_{y}=56$. Note how this expansion occurs in a way that is qualitatively different from that of Fig. 10. 


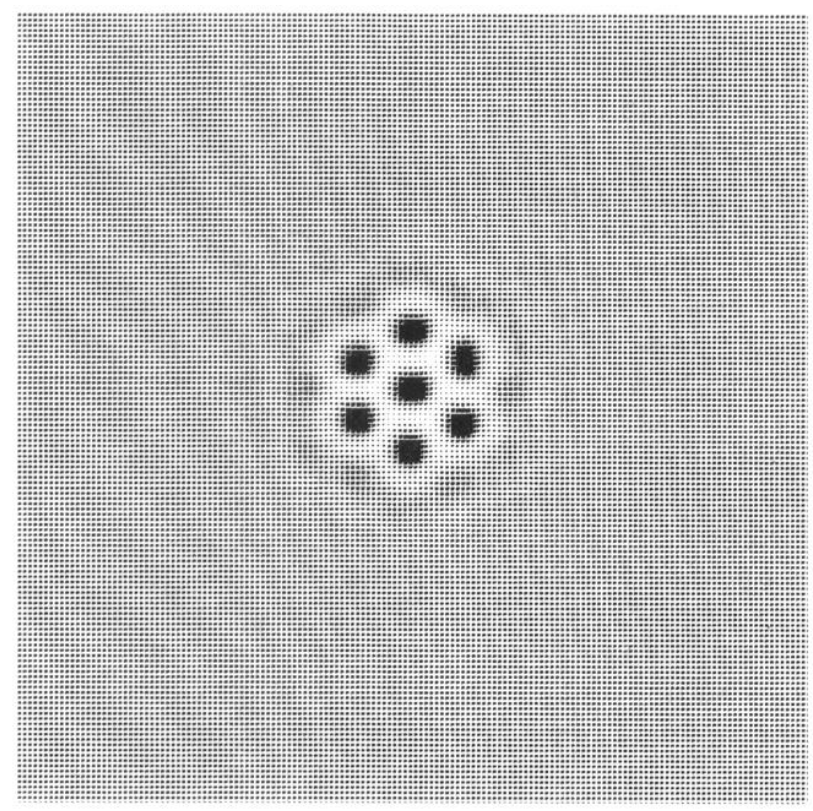

FIG. 13. Localized hexagonal structure in the LengyelEpstein model for $b=3.6$. 


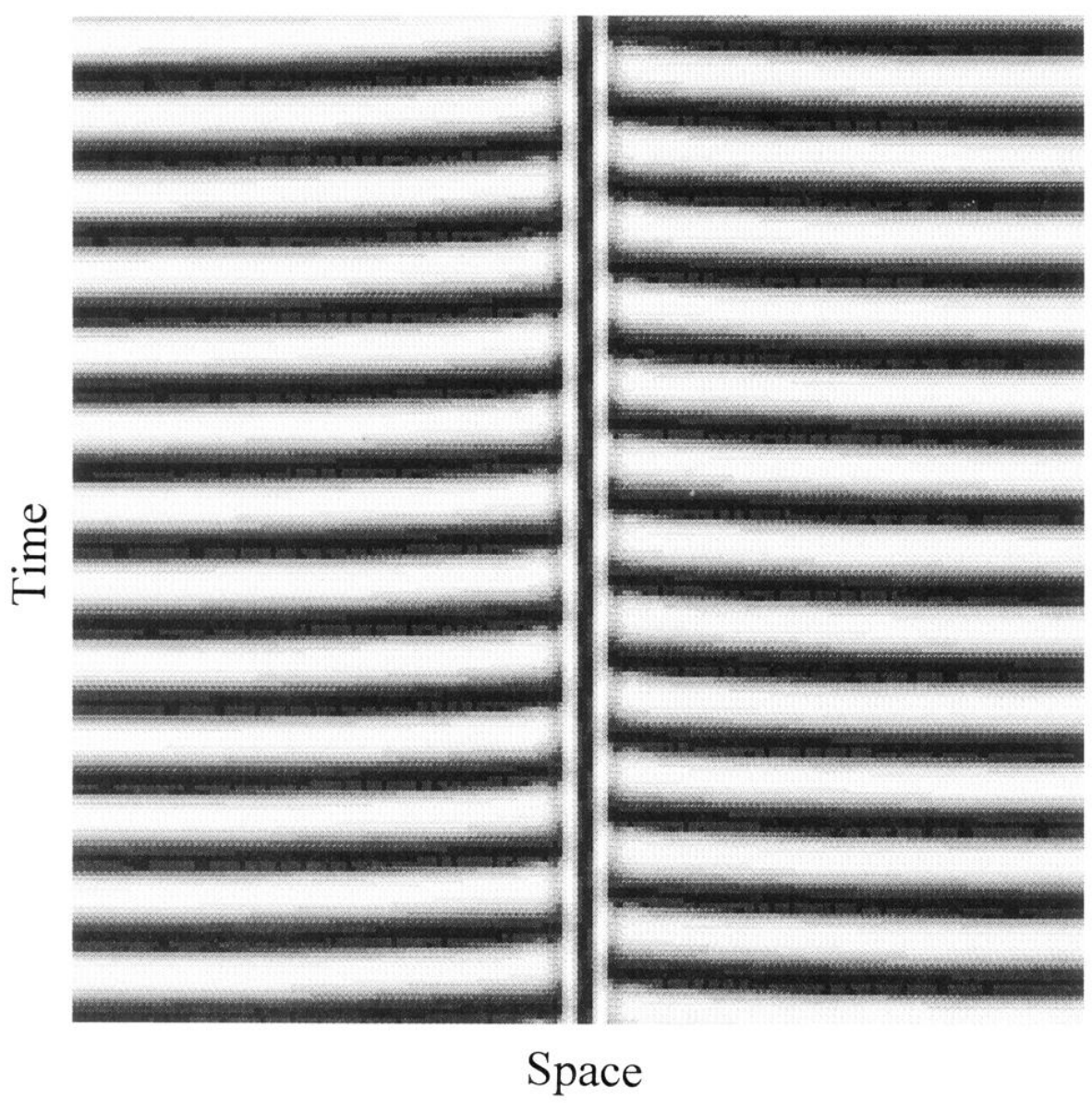

FIG. 14. Chemical flip-flop or 1D spiral in the Lengyel-Epstein model for $a=30, b=1.1$, $c=1.5$, and $\delta=8$. A 1D stripe structure is embedded in a background of nearly homogeneous oscillations. 


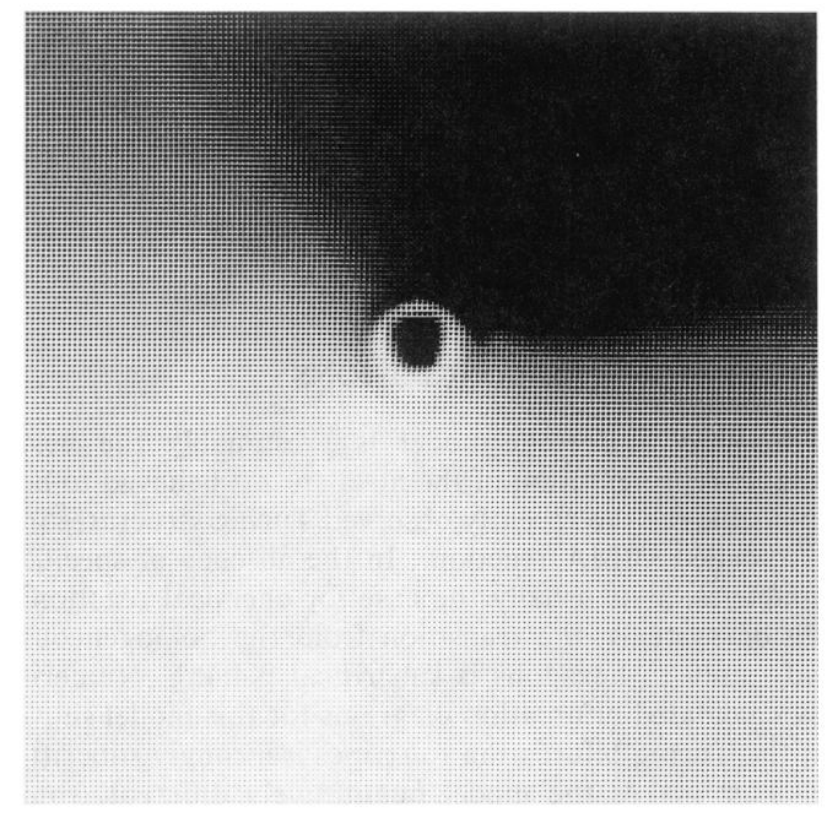

FIG. 15. 2D spiral with a Turing-induced core. Parameters are the same as Fig. 14. The rotation of the spiral is clockwise. For symmetry reasons, a similar spiral rotating in the opposite direction must also exist. 

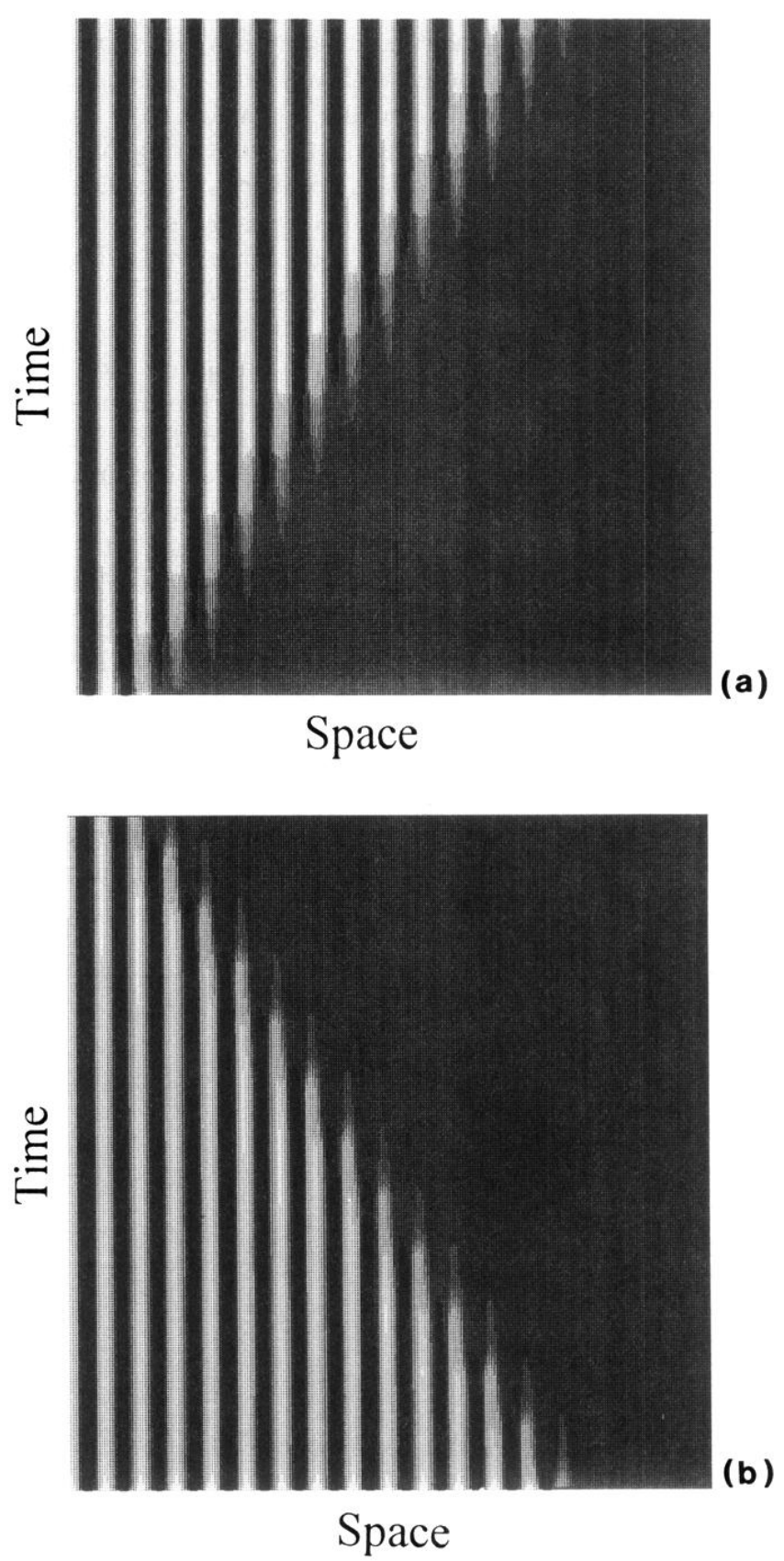

(b)

FIG. 5. Numerically computed solutions to the LengyelEpstein model with one space dimension: (a) a stripe structure invades the homogeneous steady state for $a=30$ and $b=3.0$, and (b) the homogeneous steady state invades a region with stripe structure for $a=30$ and $b=3.6$. 